The Astrophysical Journal, 235: 452-469, 1980 January 15

(C) 1980. The American Astronomical Society. All rights reserved. Printed in U.S.A.

\title{
THE SPREAD IN CO ABSORPTION AND EFFECTIVE TEMPERATURE AMONG THE GIANTS IN OMEGA CENTAURI
}

\author{
S. E. Persson \\ Hale Observatories, ${ }^{1}$ Carnegie Institution of Washington \\ JAY A. FrogeL \\ Cerro Tololo Inter-American Observatory ${ }^{2}$ \\ J. G. COHEN \\ Hale Observatories, ${ }^{1}$ California Institute of Technology \\ M. Aaronson \\ Steward Observatory \\ AND \\ K. MatThews \\ Hale Observatories, ${ }^{1}$ California Institute of Technology \\ Received 1979 April 9; accepted 1979 July 31
}

\begin{abstract}
Infrared photometric observations of 82 stars on the upper giant branch of $\omega$ Centaur1 are presented. The data show that $\omega$ Cen differs markedly from other globular clusters studied in the infrared in two respects. First, at a given $V-K$ color, there is a wide spread in CO absorption $(\gtrsim 0.1 \mathrm{mag})$ in the $3 \mathrm{mag}$ interval below the red giant tip; this range is approximately 5 times that found for other clusters. Second, at a given luminosity, $V-K$ has a spread of up to 1 mag; this range is also much larger than that found for other clusters. The latter result leads us to conclude, in agreement with recent findings by other authors, that there are star-to-star variations in heavymetal abundance within $\omega$ Cen of a factor of 30 or more.

Comparison of the spread in $\mathrm{CO}$ absorption with that in $V-K$ reveals two sequences of stars, one which has "enhanced" CO absorption and one which has "normal" CO absorption when compared to the run of $\mathrm{CO}$ with $V-K$ among stars in other globular clusters. The proportion of stars having "enhanced" CO exceeds $50 \%$. Many of the strong CN stars in the cluster also have "enhanced" CO. Explanations for the origin of these sequences which involve primordial abundance variations as well as mixing phenomena are discussed briefly. Implications for the identification of the "second parameter" as the CNO abundance are also discussed.

Effective temperatures and bolometric luminosities are given for the stars.

Subject headings: clusters: globular - stars: abundances — stars: evolution — stars: late-type
\end{abstract}

\section{INTRODUCTION}

The giant-branch stars of the globular cluster $\omega$ Centauri display striking peculiarities in their photometric indices and derived surface abundances which set this cluster apart from all other well-studied globulars. The major photometric anomaly is that the giant branch of $\omega$ Cen is considerably wider in $B-V$ than that of any other globular cluster (Wooley et al. 1966; Dickens and Woolley 1967; Cannon and Stobie 1973). Other peculiarities include the membership of three carbon stars (Harding 1962; Dickens 1972; Wing and Stock 1973; Bond 1975), a number of red variables (Martin 1938; Dickens, Feast, and Lloyd-Evans 1972; Feast 1973; Wing 1973), and stars showing clear evidence for surface enrichment of carbon, nitrogen,

${ }^{1}$ The Hale Observatories are operated jointly by the Carnegie Institution of Washington and the California Institute of Technology.

${ }^{2}$ Cerro Tololo Inter-American Observatory is supported by the National Science Foundation under contract AST 78-27879. and $s$-process elements (e.g., Bessell and Norris 1976; Dickens and Bell 1976; Mallia 1976; Norris and Bessell 1975, 1977).

Two hypotheses have been advanced to account qualitatively for these anomalies. In the "primordial" hypothesis, the cluster is postulated to contain stars spread over a substantial range in $[\mathrm{Fe} / \mathrm{H}]$, and this directly produces the spread in $B-V$ at a given luminosity. In the "mixing" hypothesis, material which has undergone nuclear processing in the stellar interior is brought to the surface. The elements primarily affected are $\mathrm{C}, \mathrm{N}$, and $\mathrm{O}$, and the mixing process is postulated to occur to a widely varying degree among the stars of the cluster. The result is that the amount of absorption due to $\mathrm{CN}, \mathrm{CH}$, and $\mathrm{CO}$ is highly variable from star to star, and this is supposed to affect the broad-band colors to the extent that no range in $\mathrm{Fe} / \mathrm{H}$ is required to explain the wide giant branch. Recent demonstrations by Freeman and Rodgers $(1975,1978)$ and Butler, Dickens, and Epps 
(1978) of a spread of roughly one order of magnitude in heavy-metal abundance among the RR Lyrae variables, and by Norris (1978), Cohen (1978), and Lloyd-Evans (1978) of the existence of variations in heavy-element abundances among the giants, argue strongly for the "primordial" hypothesis. It seems unlikely, however, that a spread in the primordial abundances of the heavy elements can by itself explain the CN strong stars and the carbon stars or account for the unequivocal evidence for surface enrichment of $s$-process elements in some stars (Bell and Dickens 1974; Dickens and Bell 1976). Thus it seems reasonable to suppose that both "mixing" and "primordial" abundance variations may be required.

In this paper we present and discuss infrared data for 82 giants in $\omega$ Cen. Our aims in obtaining these data were twofold. First, we wished to obtain accurate measurements of the strength of the $\mathrm{CO}$ band in order to examine the dependence of the $\mathrm{CO}$ absorption on temperature for the $\omega$ Cen stars and to compare this dependence with those found for normal clusters. Second, we wished to determine the intrinsic spread of the giant branch in effective temperature at a given bolometric luminosity. This spread can be used as an independent measure of the spread in heavy-metal abundance (Rood 1978; Renzini 1977). The values of effective temperature are derived from the $V-K$ colors by a transformation which is nearly independent of metal abundance or surface gravity (Cohen, Frogel, and Persson 1978, hereafter CFP).

The observations are presented in $\S \mathrm{II}$, and the most important observational results are given in § III. In $\S$ IV we examine the spread in indices and colors on the giant branch and discuss the principal results. The derived physical parameters $T_{\text {eff }}$ and $L_{\text {bol }}$ are presented in $\S \mathrm{V}$, and a summary is given in $\S \mathrm{VI}$. Several twocolor diagrams are presented and discussed in an Appendix.

Some of the results and conclusions of this paper were presented at the NATO Advanced Study Institute on Globular Clusters (Persson and Frogel 1978).

\section{OBSERVATIONS}

\section{a) Infrared Photometry}

The observations were made using the $2.5 \mathrm{~m}$ $\mathrm{Du}$ Pont telescope of the Las Campanas Observatory and the $4.0 \mathrm{~m}$ telescope of Cerro Tololo InterAmerican Observatory in 1978 March and July and 1979 February. Two InSb detector systems were used to make broad-band $J, H$, and $K$ measurements (1.25, 1.65 , and $2.2 \mu \mathrm{m}$, respectively) and narrow-band $\mathrm{CO}$ and $\mathrm{H}_{2} \mathrm{O}$ index measurements $(2.20-2.36 \mu \mathrm{m}$ and $2.20-2.00 \mu \mathrm{m}$ colors, respectively), as described in CFP. ${ }^{3}$ The aperture diameters used varied between

${ }^{3}$ The data have been transformed to the system of Frogel et al. (1978) and Aaronson, Frogel, and Persson (1978). The transformation uncertainties are less than $0.03 \mathrm{mag}$ in the broad-band colors and magnitudes and less than $0.015 \mathrm{mag}$ in the narrow-band indices. A detailed discussion of these transformations will be given separately. The data are on the same system as those presented in CFP and Frogel, Persson, and Cohen (1979). $5^{\prime \prime}$ and $15^{\prime \prime}$, and care was taken to avoid background stars in signal and sky apertures.

Many of the stars observed were taken from the photometrically unbiased sample of Cannon and Stobie (1973); no definite or probable field stars were included. A few stars known to be spectroscopically peculiar (R. Zinn, private communication) and several fainter stars on the lower giant branch in Geyer's (1967) sample and from the ROA catalog (Woolley et al. 1966) were also observed. We included some of the stars shown by Lloyd-Evans $(1977 a, b)$ to lie far to the red of the bulk of the giant-branch stars in a diagram of $I_{\mathrm{K}}$ versus $V-I_{\mathrm{K}}$ (where $I_{\mathrm{K}}$ denotes $I$ on the Kron system) and shown by him to be cluster members. Finally, of the variables studied by Dickens, Feast, and Lloyd-Evans (1972) we measured those which lie in relatively uncrowded regions.

Table 1 lists the observational data and also the colors corrected for reddening corresponding to $E(B-V)=0.11$ (e.g., Cannon and Stobie 1973). The reddening corrections amount to $0.03,0.31,0.03$, $0.02,0.005$, and $-0.005 \mathrm{mag}$ for $K, V-K, J-H$, $H-K, \mathrm{CO}$ index, and $\mathrm{H}_{2} \mathrm{O}$ index, respectively. Throughout the rest of this paper colors corrected for reddening are noted with a subscript zero, except for the $\mathrm{CO}$ and $\mathrm{H}_{2} \mathrm{O}$ indices. We have included in Table 1 the available $U B V$ data corrected for reddening. The $(B-V)_{0}$ colors from the ROA catalog have been decreased by $0.07 \mathrm{mag}$ in accordance with the results of Cannon and Stobie (1973).

Unfortunately, we were able to repeat the measurements of only seven stars. Repeated measurements of these stars (including seven measurement of ROA 70) agreed to within the uncertainties given at the bottom of Table 1. Our photometry overlaps that of Glass and Feast $(1973,1977)$, and we can compare the $J H K$ measurements of 18 stars. Of these we exclude V6, V17, and ROA 320, which vary strongly in the infrared as shown by Glass and Feast. The agreement is generally satisfactory both in magnitudes and in colors, considering the differences in the photometric systems.

$$
\text { b) }(V-K)_{0} \text { Colors }
$$

The $(V-K)_{0}$ colors listed in Table 1 were formed from the $V$ data of Cannon and Stobie (1973) whenever possible. For the nonvariable stars having only photographic data (ROA catalog), we adjusted the ROA $V$ magnitudes fainter by $0.04 \mathrm{mag}$ in accordance with the average difference between the ROA and photoelectric values found by Cannon and Stobie (1973). The $(V-K)_{0}$ colors for the stars having only photographic data are probably uncertain by at least $0.05 \mathrm{mag}$.

Several of the Cannon and Stobie (1973) stars were noted by them to be mildly variable, and for these the uncertainties in $(V-K)_{0}$ are $\sim 0.10 \mathrm{mag}$. For the larger-amplitude irregular variables studied by Martin (1938) and Dickens et al., the $(V-K)_{0}$ values are more uncertain, as noted in Table 1 . Two procedures were adopted to give indicative $(V-K)_{0}$ values for the larger-amplitude variables. First, for V6, V17, and 
TABLE 1

$\omega$ Centauri Photometry

\begin{tabular}{|c|c|c|c|c|c|c|c|c|c|c|c|c|}
\hline \multirow{2}{*}{ Star ${ }^{\dagger}$} & \multicolumn{3}{|c|}{ Observed* } & \multicolumn{8}{|c|}{ Reddening Corrected } & \multirow[b]{2}{*}{ Notes } \\
\hline & $\mathbf{K}$ & $\mathrm{J}-\mathrm{H}$ & $\mathrm{H}-\mathrm{K}$ & $\mathbf{K}_{0}$ & $(U-V)_{0}$ & $(B-V)_{0}$ & $(\mathrm{~V}-\mathrm{K})_{0}$ & $(\mathrm{~J}-\mathrm{H})_{0}$ & $(\mathrm{H}-\mathrm{K})_{0}$ & $\mathrm{CO}$ & $\mathrm{H}_{2} \mathrm{O}$ & \\
\hline $\begin{array}{l}40 \\
43 \\
46 \\
48 \\
49\end{array}$ & $\begin{array}{l}7.92 \\
7.73 \\
7.82 \\
7.80 \\
7.92\end{array}$ & $\begin{array}{l}0.67 \\
0.79 \\
0.70 \\
0.71 \\
0.72\end{array}$ & $\begin{array}{l}0.15 \\
0.17 \\
0.16 \\
0.16 \\
0.13\end{array}$ & $\begin{array}{l}7.89 \\
7.70 \\
7.79 \\
7.77 \\
7.89\end{array}$ & $\begin{array}{l}2.72 \\
3.36 \\
2.94 \\
2.81 \\
2.81\end{array}$ & $\begin{array}{l}1.39 \\
1.60 \\
1.47 \\
1.45 \\
1.48\end{array}$ & $\begin{array}{l}3.14 \\
3.60 \\
3.41 \\
3.40 \\
3.34\end{array}$ & $\begin{array}{l}0.64 \\
0.76 \\
0.67 \\
0.68 \\
0.69\end{array}$ & $\begin{array}{l}0.13 \\
0.15 \\
0.14 \\
0.14 \\
0.11\end{array}$ & $\begin{array}{l}0.095 \\
0.140 \\
0.040 \\
0.040 \\
0.060\end{array}$ & $\begin{array}{l}0.020 \\
0.070 \\
0.035 \\
0.030 \\
0.055\end{array}$ & $\begin{array}{l}M \\
M, \operatorname{var} \\
M \\
M \\
M, \operatorname{var}\end{array}$ \\
\hline $\begin{array}{l}53 \\
55 \\
56 \\
58 \\
61\end{array}$ & $\begin{array}{l}7.65 \\
7.66 \\
7.86 \\
8.19 \\
7.75\end{array}$ & $\begin{array}{l}0.73 \\
0.68 \\
0.73 \\
0.65 \\
0.70\end{array}$ & $\begin{array}{l}0.17 \\
0.22 \\
0.15 \\
0.15 \\
0.14\end{array}$ & $\begin{array}{l}7.62 \\
7.63 \\
7.83 \\
8.16 \\
7.72\end{array}$ & $\begin{array}{l}3.24 \\
2.79 \\
3.19 \\
2.41 \\
3.14\end{array}$ & $\begin{array}{l}1.59 \\
1.57 \\
1.54 \\
1.31 \\
1.54\end{array}$ & $\begin{array}{l}3.62 \\
3.49 \\
3.53 \\
3.14 \\
3.48\end{array}$ & $\begin{array}{l}0.70 \\
0.65 \\
0.70 \\
0.62 \\
0.67\end{array}$ & $\begin{array}{l}0.15 \\
0.20 \\
0.13 \\
0.13 \\
0.12\end{array}$ & $\begin{array}{l}0.105 \\
0.060 \\
0.060 \\
0.020 \\
0.050\end{array}$ & $\begin{array}{l}0.035 \\
0.050 \\
0.015 \\
0.030 \\
0.055\end{array}$ & $\begin{array}{l}\text { M, var } \\
\text { M, } \\
\text { M, var } \\
\text { M, } \\
\text { m, var }\end{array}$ \\
\hline $\begin{array}{l}62 \\
70 \\
74 \\
84 \\
90\end{array}$ & $\begin{array}{l}\text { 7. } 76 \\
7.88 \\
8.40 \\
7.88 \\
7.89\end{array}$ & $\begin{array}{l}0.78 \\
0.71 \\
0.67 \\
0.74 \\
0.75\end{array}$ & $\begin{array}{l}0.16 \\
0.17 \\
0.13 \\
0.17 \\
0.15\end{array}$ & $\begin{array}{l}7.73 \\
7.85 \\
8.37 \\
7.85 \\
7.86\end{array}$ & $\begin{array}{l}3.17 \\
3.29 \\
2.31 \\
3.26 \\
3.11\end{array}$ & $\begin{array}{l}1.54 \\
1.69 \\
1.25 \\
1.55 \\
1.53\end{array}$ & $\begin{array}{l}\text { 3. } 43 \\
\text { 3. } 42 \\
\text { 3. } 03 \\
\text { 3. } 68 \\
\text { 3. } 46\end{array}$ & $\begin{array}{l}0.75 \\
0.68 \\
0.64 \\
0.71 \\
0.72\end{array}$ & $\begin{array}{l}0.14 \\
0.15 \\
0.11 \\
0.15 \\
0.13\end{array}$ & $\begin{array}{l}0.070 \\
0.150 \\
0.035 \\
0.180 \\
0.045\end{array}$ & $\begin{array}{l}0.065 \\
0.050 \\
0.040 \\
0.050 \\
0.030\end{array}$ & $\begin{array}{l}\mathrm{M} \\
\mathrm{M} \\
\mathrm{M} \\
\mathrm{M} \\
\mathrm{M}, \mathrm{var}\end{array}$ \\
\hline $\begin{array}{r}91 \\
96 \\
102 \\
124 \\
132\end{array}$ & $\begin{array}{l}8.41 \\
8.19 \\
8.20 \\
8.43 \\
7.87\end{array}$ & $\begin{array}{l}0.64 \\
0.70 \\
0.67 \\
0.69 \\
0.80\end{array}$ & $\begin{array}{l}0.14 \\
0.15 \\
0.14 \\
0.14 \\
0.16\end{array}$ & $\begin{array}{l}8.38 \\
8.16 \\
8.17 \\
8.40 \\
7.84\end{array}$ & $\begin{array}{l}2.44 \\
2.69 \\
2.60 \\
2.44 \\
. .\end{array}$ & $\begin{array}{l}1.28 \\
1.37 \\
1.35 \\
1.27 \\
1.53\end{array}$ & $\begin{array}{l}3.14 \\
3.26 \\
3.17 \\
3.09 \\
3.72\end{array}$ & $\begin{array}{l}0.61 \\
0.67 \\
0.64 \\
0.66 \\
0.77\end{array}$ & $\begin{array}{l}0.12 \\
0.13 \\
0.12 \\
0.12 \\
0.14\end{array}$ & $\begin{array}{l}0.015 \\
0.050 \\
0.015 \\
0.060 \\
0.155\end{array}$ & $\begin{array}{l}0.010 \\
0.045 \\
0.030 \\
0.050 \\
0.100\end{array}$ & $\begin{array}{l}\mathrm{M} \\
\mathrm{M} \\
\mathrm{M} \\
\mathrm{M} \\
\mathrm{M}, 2 \text {, var }\end{array}$ \\
\hline $\begin{array}{l}139 \\
150 \\
155 \\
159 \\
161\end{array}$ & $\begin{array}{l}8.49 \\
8.17 \\
8.58 \\
8.73 \\
8.63\end{array}$ & $\begin{array}{l}0.68 \\
0.75 \\
0.68 \\
0.63 \\
0.67\end{array}$ & $\begin{array}{l}0.15 \\
0.16 \\
0.15 \\
0.13 \\
0.13\end{array}$ & $\begin{array}{l}8.46 \\
8.14 \\
8.55 \\
8.70 \\
8.60\end{array}$ & $\begin{array}{l}3.41 \\
2.48 \\
2.17 \\
2.44\end{array}$ & $\begin{array}{l}1.41 \\
1.59 \\
1.31 \\
1.21 \\
1.28\end{array}$ & $\begin{array}{l}3.17 \\
3.53 \\
3.11 \\
2.97 \\
3.03\end{array}$ & $\begin{array}{l}0.65 \\
0.72 \\
0.65 \\
0.60 \\
0.64\end{array}$ & $\begin{array}{l}0.13 \\
0.14 \\
0.13 \\
0.11 \\
0.11\end{array}$ & $\begin{array}{l}0.135 \\
0.070 \\
0.075 \\
0.040 \\
0.070\end{array}$ & $\begin{array}{l}0.040 \\
0.045 \\
0.030 \\
0.000 \\
0.035\end{array}$ & $\begin{array}{l}\mathrm{m}, 1 \\
\mathrm{M} \\
\mathrm{M} \\
\mathrm{M} \\
\mathrm{m}\end{array}$ \\
\hline $\begin{array}{l}162 \\
171 \\
179 \\
180 \\
201\end{array}$ & $\begin{array}{l}8.31 \\
8.48 \\
8.00 \\
8.96 \\
7.95\end{array}$ & $\begin{array}{l}0.76 \\
0.71 \\
0.77 \\
0.66 \\
0.75\end{array}$ & $\begin{array}{l}0.16 \\
0.14 \\
0.21 \\
0.12 \\
0.24\end{array}$ & $\begin{array}{l}8.28 \\
8.45 \\
7.97 \\
8.93 \\
7.92\end{array}$ & $\begin{array}{l}3.40 \\
2.75 \\
2.00 \\
. .\end{array}$ & $\begin{array}{l}1.59 \\
1.37 \\
1.53 \\
1.18 \\
1.43\end{array}$ & $\begin{array}{l}3.52 \\
3.28 \\
3.76 \\
2.76 \\
3.88\end{array}$ & $\begin{array}{l}0.73 \\
0.68 \\
0.74 \\
0.63 \\
0.72\end{array}$ & $\begin{array}{l}0.14 \\
0.12 \\
0.19 \\
0.10 \\
0.22\end{array}$ & $\begin{array}{l}0.080 \\
0.145 \\
0.195 \\
0.035 \\
0.230\end{array}$ & $\begin{array}{l}0.050 \\
0.035 \\
0.065 \\
0.060 \\
0.090\end{array}$ & $\begin{array}{l}\text { M, var } \\
\text { m } \\
M, 2 \\
m, 1 \\
M, 2\end{array}$ \\
\hline $\begin{array}{l}213 \\
219 \\
231 \\
234 \\
248\end{array}$ & $\begin{array}{l}9.24 \\
8.46 \\
8.47 \\
9.24 \\
8.24\end{array}$ & $\begin{array}{l}0.60 \\
0.74 \\
0.79 \\
0.57 \\
0.83\end{array}$ & $\begin{array}{l}0.11 \\
0.17 \\
0.18 \\
0.12 \\
0.17\end{array}$ & $\begin{array}{l}9.21 \\
8.43 \\
8.44 \\
9.21 \\
8.21\end{array}$ & $\begin{array}{l}1.67 \\
3.05 \\
\text { i. } 77 \\
. . \quad\end{array}$ & $\begin{array}{l}1.03 \\
1.47 \\
1.45 \\
1.05 \\
1.66\end{array}$ & $\begin{array}{l}2.67 \\
3.43 \\
3.45 \\
2.70 \\
3.72\end{array}$ & $\begin{array}{l}0.57 \\
0.71 \\
0.76 \\
0.54 \\
0.80\end{array}$ & $\begin{array}{l}0.09 \\
0.15 \\
0.16 \\
0.10 \\
0.15\end{array}$ & $\begin{array}{r}0.010 \\
0.180 \\
0.080 \\
-0.010 \\
0.100\end{array}$ & $\begin{array}{l}0.025 \\
0.060 \\
0.040 \\
0.005 \\
0.060\end{array}$ & $\begin{array}{l}M \\
M \\
M, 2 \\
M \\
M, 2\end{array}$ \\
\hline $\begin{array}{l}253 \\
256 \\
269 \\
270 \\
272\end{array}$ & $\begin{array}{l}9.10 \\
9.19 \\
9.25 \\
8.85 \\
9.11\end{array}$ & $\begin{array}{l}0.63 \\
0.62 \\
0.63 \\
0.70 \\
0.66\end{array}$ & $\begin{array}{l}0.13 \\
0.14 \\
0.13 \\
0.15 \\
0.13\end{array}$ & $\begin{array}{l}9.07 \\
9.16 \\
9.22 \\
8.82 \\
9.08\end{array}$ & $\begin{array}{l}2.46 \\
\text { i. } .89 \\
2.77 \\
2.36\end{array}$ & $\begin{array}{l}1.26 \\
1.06 \\
1.11 \\
1.39 \\
1.25\end{array}$ & $\begin{array}{l}2.93 \\
2.80 \\
2.79 \\
3.26 \\
2.96\end{array}$ & $\begin{array}{l}0.60 \\
0.59 \\
0.60 \\
0.67 \\
0.63\end{array}$ & $\begin{array}{l}0.11 \\
0.12 \\
0.11 \\
0.13 \\
0.11\end{array}$ & $\begin{array}{l}0.005 \\
0.000 \\
0.025 \\
0.200 \\
0.085\end{array}$ & $\begin{array}{r}0.005 \\
-0.015 \\
0.045 \\
0.055 \\
0.055\end{array}$ & $\begin{array}{l}\mathrm{M} \\
\mathrm{m}, 2 \\
\mathrm{~m} \\
\mathrm{~m} \\
\mathrm{~m}\end{array}$ \\
\hline $\begin{array}{l}279 \\
287 \\
297 \\
300 \\
312\end{array}$ & $\begin{array}{l}9.20 \\
9.09 \\
9.28 \\
8.47 \\
9.23\end{array}$ & $\begin{array}{l}0.62 \\
0.65 \\
0.63 \\
0.87 \\
0.66\end{array}$ & $\begin{array}{l}0.15 \\
0.12 \\
0.13 \\
0.15 \\
0.14\end{array}$ & $\begin{array}{l}9.17 \\
9.06 \\
9.25 \\
8.44 \\
9.20\end{array}$ & $\begin{array}{l}2.27 \\
2.51 \\
2.14 \\
2.07\end{array}$ & $\begin{array}{l}1.27 \\
1.31 \\
1.20 \\
1.40 \\
1.19\end{array}$ & $\begin{array}{l}2.81 \\
3.01 \\
2.85 \\
3.62 \\
2.90\end{array}$ & $\begin{array}{l}0.59 \\
0.62 \\
0.60 \\
0.84 \\
0.63\end{array}$ & $\begin{array}{l}0.13 \\
0.10 \\
0.11 \\
0.13 \\
0.12\end{array}$ & $\begin{array}{l}0.105 \\
0.150 \\
0.085 \\
0.155 \\
0.065\end{array}$ & $\begin{array}{l}0.035 \\
0.020 \\
0.040 \\
0.050 \\
0.010\end{array}$ & $\begin{array}{l}\mathrm{m} \\
\mathrm{M} \\
\mathrm{m} \\
\mathrm{M}, 2 \\
\mathrm{~m}\end{array}$ \\
\hline $\begin{array}{l}357 \\
364 \\
371 \\
394 \\
402\end{array}$ & $\begin{array}{l}8.76 \\
9.62 \\
8.92 \\
9.43 \\
9.84\end{array}$ & $\begin{array}{l}0.81 \\
0.59 \\
0.77 \\
0.65 \\
0.54\end{array}$ & $\begin{array}{l}0.16 \\
0.14 \\
0.15 \\
0.13 \\
0.12\end{array}$ & $\begin{array}{l}8.73 \\
9.59 \\
8.89 \\
9.40 \\
9.81\end{array}$ & $\begin{array}{l}1.55 \\
3.17 \\
2.35 \\
1.56\end{array}$ & $\begin{array}{l}1.30 \\
1.00 \\
1.49 \\
1.24 \\
0.96\end{array}$ & $\begin{array}{l}3.47 \\
2.57 \\
3.45 \\
2.92 \\
2.53\end{array}$ & $\begin{array}{l}0.78 \\
0.56 \\
0.74 \\
0.62 \\
0.51\end{array}$ & $\begin{array}{l}0.14 \\
0.12 \\
0.13 \\
0.11 \\
0.10\end{array}$ & $\begin{array}{l}0.130 \\
0.000 \\
0.105 \\
0.105 \\
0.005\end{array}$ & $\begin{array}{l}0.030 \\
0.010 \\
0.055 \\
0.005 \\
0.035\end{array}$ & $\begin{array}{l}\text { M, } 2 \\
M \\
M, \operatorname{var} \\
M \\
M\end{array}$ \\
\hline $\begin{array}{l}415 \\
421 \\
425 \\
447 \\
451\end{array}$ & $\begin{array}{l}9.85 \\
9.38 \\
8.42 \\
8.53 \\
9.16\end{array}$ & $\begin{array}{l}0.56 \\
0.71 \\
0.82 \\
0.84 \\
0.75\end{array}$ & $\begin{array}{l}0.12 \\
0.17 \\
0.22 \\
0.21 \\
0.18\end{array}$ & $\begin{array}{l}9.82 \\
9.35 \\
8.39 \\
8.50 \\
9.13\end{array}$ & $\begin{array}{l}1.51 \\
\therefore . \\
. \\
.\end{array}$ & $\begin{array}{l}0.99 \\
1.32 \\
1.51: \\
1.53 \\
1.35\end{array}$ & $\begin{array}{l}2.50 \\
3.02 \\
\text { 3. } 99: \\
\text { 3. } 94 \\
\text { 3. } 31\end{array}$ & $\begin{array}{l}0.53 \\
0.68 \\
0.79 \\
0.81 \\
0.72\end{array}$ & $\begin{array}{l}0.10 \\
0.15 \\
0.20 \\
0.19 \\
0.16\end{array}$ & $\begin{array}{l}0.015 \\
0.060 \\
0.155 \\
0.110 \\
0.110\end{array}$ & $\begin{array}{l}0.005 \\
0.035 \\
0.060 \\
0.030 \\
0.030\end{array}$ & $\begin{array}{l}\mathrm{m} \\
\mathrm{M}, 2 \\
\mathrm{M}, 2 \\
\mathrm{M}, 2 \\
\mathrm{M}, 2\end{array}$ \\
\hline
\end{tabular}


TABLE 1-Continued

\begin{tabular}{|c|c|c|c|c|c|c|c|c|c|c|c|c|}
\hline \multirow[b]{2}{*}{$\operatorname{Star}^{+}$} & \multicolumn{3}{|c|}{ Observed* } & \multicolumn{8}{|c|}{ Reddening Corrected } & \multirow[b]{2}{*}{ Notes } \\
\hline & $\mathrm{K}$ & $\mathrm{J}-\mathrm{H}$ & $\mathrm{H}-\mathrm{K}$ & $\mathbf{K}_{0}$ & $(U-V)_{0}$ & $(B-V)_{0}$ & $(\mathrm{~V}-\mathrm{K})_{0}$ & $(\mathrm{~J}-\mathrm{H})_{0}$ & $(\mathrm{H}-\mathrm{K})_{0}$ & $\mathrm{CO}$ & $\mathrm{H}_{2} \mathrm{O}$ & \\
\hline $\begin{array}{l}461 \\
462 \\
464 \\
465 \\
472\end{array}$ & $\begin{array}{r}9.88 \\
10.05 \\
9.89 \\
9.62 \\
9.66\end{array}$ & $\begin{array}{l}0.63 \\
0.53 \\
0.59 \\
0.66 \\
0.65\end{array}$ & $\begin{array}{l}0.12 \\
0.11 \\
0.09 \\
0.14 \\
0.16\end{array}$ & $\begin{array}{r}9.85 \\
10.02 \\
9.86 \\
9.59 \\
9.63\end{array}$ & $\begin{array}{l}1.36 \\
1.81 \\
. \quad .\end{array}$ & $\begin{array}{l}1.05 \\
0.90 \\
1.07 \\
1.12 \\
1.22\end{array}$ & $\begin{array}{l}2.61 \\
2.40 \\
2.65 \\
2.87 \\
2.84\end{array}$ & $\begin{array}{l}0.60 \\
0.50 \\
0.56 \\
0.63 \\
0.62\end{array}$ & $\begin{array}{l}0.10 \\
0.09 \\
0.07 \\
0.12 \\
0.14\end{array}$ & $\begin{array}{l}0.055 \\
\dot{0} . \dot{0} \dot{0} \\
0.050 \\
0.135\end{array}$ & $\begin{array}{c}\ldots \\
\dot{0} .045 \\
\therefore \\
\therefore\end{array}$ & $\begin{array}{l}\mathrm{m}, 2 \\
\mathrm{M} \\
\mathrm{m} \\
2 \\
2\end{array}$ \\
\hline $\begin{array}{l}480 \\
483 \\
505 \\
509 \\
513\end{array}$ & $\begin{array}{r}9.70 \\
9.95 \\
9.69 \\
10.10 \\
8.58\end{array}$ & $\begin{array}{l}0.68 \\
0.55 \\
0.68 \\
0.54 \\
0.88\end{array}$ & $\begin{array}{l}0.16 \\
0.13 \\
0.14 \\
0.12 \\
0.19\end{array}$ & $\begin{array}{r}9.67 \\
9.92 \\
9.66 \\
10.07 \\
8.55\end{array}$ & $\begin{array}{l}\text { i. } 47 \\
\text { i. } 37\end{array}$ & $\begin{array}{l}1.33 \\
0.96 \\
1.10 \\
0.89 \\
1.49\end{array}$ & $\begin{array}{l}2.80 \\
2.56 \\
2.87 \\
2.53 \\
3.99\end{array}$ & $\begin{array}{l}0.65 \\
0.52 \\
0.65 \\
0.51 \\
0.85\end{array}$ & $\begin{array}{l}0.14 \\
0.11 \\
0.12 \\
0.10 \\
0.17\end{array}$ & $\begin{array}{l}0.060 \\
0.015 \\
0.045 \\
0.145\end{array}$ & $\begin{array}{l}0.020 \\
0.000 \\
\vdots \\
0.060\end{array}$ & $\begin{array}{l}\mathrm{m}:, 2 \\
\mathrm{~m} \\
\mathrm{~m}, 2 \\
\mathrm{M} \\
\mathrm{M}, 2\end{array}$ \\
\hline $\begin{array}{r}537 \\
557 \\
577 \\
3598 \\
5941\end{array}$ & $\begin{array}{r}9.85 \\
9.83 \\
9.97 \\
11.84 \\
11.03\end{array}$ & $\begin{array}{l}0.65 \\
0.66 \\
0.59 \\
0.50 \\
0.49\end{array}$ & $\begin{array}{l}0.11 \\
0.15 \\
0.16 \\
0.04 \\
0.07\end{array}$ & $\begin{array}{r}9.82 \\
9.80 \\
9.94 \\
11.81 \\
11.00\end{array}$ & $\begin{array}{l}1.90 \\
\cdot . \\
0.99 \\
1.01\end{array}$ & $\begin{array}{l}1.13 \\
1.24 \\
1.37 \\
0.76 \\
0.77\end{array}$ & $\begin{array}{l}2.74 \\
2.82 \\
2.71 \\
2.21 \\
2.16\end{array}$ & $\begin{array}{l}0.62 \\
0.63 \\
0.56 \\
0.47 \\
0.46\end{array}$ & $\begin{array}{l}0.09 \\
0.13 \\
0.14 \\
0.02 \\
0.05\end{array}$ & $\begin{array}{l}0.100 \\
0.055 \\
0.120 \\
. . \\
. .\end{array}$ & $\begin{array}{l}0.025 \\
\dot{0} .035 \\
\therefore . \\
\therefore .\end{array}$ & $\begin{array}{l}\mathrm{M} \\
\mathrm{m}, 2 \\
\mathrm{~m}: 2 \\
\mathrm{M}: \\
\mathrm{M}\end{array}$ \\
\hline $\begin{array}{r}6113 \\
\text { G55 } \\
\text { G78 } \\
\text { G318 }\end{array}$ & $\begin{array}{l}11.09 \\
11.64 \\
11.34 \\
10.62\end{array}$ & $\begin{array}{l}0.53 \\
0.54 \\
0.51 \\
0.52\end{array}$ & $\begin{array}{l}0.08 \\
0.09 \\
0.08 \\
0.10\end{array}$ & $\begin{array}{l}11.06 \\
11.61 \\
11.31 \\
10.59\end{array}$ & $\begin{array}{l}1.31 \\
0.91 \\
1.12 \\
1.07\end{array}$ & $\begin{array}{l}0.89 \\
0.83 \\
0.95 \\
0.86\end{array}$ & $\begin{array}{l}2.25 \\
2.03 \\
2.14 \\
2.40\end{array}$ & $\begin{array}{l}0.50 \\
0.51 \\
0.48 \\
0.49\end{array}$ & $\begin{array}{l}0.06 \\
0.07 \\
0.06 \\
0.08\end{array}$ & $\begin{array}{l}. \\
. \\
.\end{array}$ & $\begin{array}{l}. \\
. \\
.\end{array}$ & $\begin{array}{l}M \\
3 \\
3 \\
3\end{array}$ \\
\hline $\begin{array}{r}\text { V6 } \\
\text { V17 } \\
\text { V53 } \\
\text { V138 } \\
\text { V148 }\end{array}$ & $\begin{array}{l}7.12 \\
7.57 \\
8.17 \\
7.91 \\
7.92\end{array}$ & $\begin{array}{l}0.87 \\
0.81 \\
0.71 \\
0.66 \\
0.72\end{array}$ & $\begin{array}{l}0.32 \\
0.24 \\
0.16 \\
0.17 \\
0.18\end{array}$ & $\begin{array}{l}7.09 \\
7.54 \\
8.14 \\
7.88 \\
7.89\end{array}$ & & $\begin{array}{l}\text { 1. } 43: \\
\text { 1. } 69: \\
\text { 1. } 67: \\
\text { 1. } 30: \\
\text { 1. } 34:\end{array}$ & $\begin{array}{l}4.8 \\
4.1 \\
3.46 \\
3.23 \\
3.51\end{array}$ & $\begin{array}{l}0.84 \\
0.78 \\
0.68 \\
0.63 \\
0.69\end{array}$ & $\begin{array}{l}0.30 \\
0.22 \\
0.14 \\
0.15 \\
0.16\end{array}$ & $\begin{array}{l}0.095 \\
0.170 \\
0.085 \\
0.005 \\
0.075\end{array}$ & $\begin{array}{l}0.150 \\
0.100 \\
0.050 \\
0.010 \\
0.030\end{array}$ & $\begin{array}{l}M, 4,7 \\
M, 4,6 \\
M, 5,6 \\
5,6 \\
5,6\end{array}$ \\
\hline $\begin{array}{r}\text { V } 162 \\
\text { V } 164 \\
\text { ROA } 320\end{array}$ & $\begin{array}{l}8.12 \\
8.22 \\
7.85\end{array}$ & $\begin{array}{l}0.66 \\
0.82 \\
0.76\end{array}$ & $\begin{array}{l}0.15 \\
0.17 \\
0.23\end{array}$ & $\begin{array}{l}8.09 \\
8.19 \\
7.82\end{array}$ & & $\begin{array}{l}\text { 1. } 48: \\
\text { 1. } 76: \\
\text { 1. } 54:\end{array}$ & $\begin{array}{l}3.20 \\
3.83 \\
3.8\end{array}$ & $\begin{array}{l}0.63 \\
0.79 \\
0.73\end{array}$ & $\begin{array}{l}0.13 \\
0.15 \\
0.21\end{array}$ & $\begin{array}{l}0.010 \\
0.095 \\
0.300\end{array}$ & $\begin{array}{l}0.015 \\
0.035 \\
0.170\end{array}$ & $\begin{array}{l}\mathrm{M}, 5,6 \\
\mathrm{M}, 5,6 \\
4,7\end{array}$ \\
\hline
\end{tabular}

$\dagger$ Star numbers are from the ROA catalog except for G55, G78, and G318 (Geyer 1967) and the variables (Martin 1938).

* Observational uncertainties are typically $\pm 0.02 \mathrm{mag}$ in $J-H$ and $H-K, \pm 0.03 \mathrm{mag}$ in $V-K$ and $K, \pm 0.015 \mathrm{mag}$ in CO index, and $\pm 0.02 \mathrm{mag}$ in $\mathrm{H}_{2} \mathrm{O}$ index unless noted otherwise.

Notes

M Radial velocity member (Cannon and Stobie 1973; Dickens et al. 1972; Lloyd-Evans 1977a, b; Norris and Bessell 1977).

m Proper motion member (ROA catalog).

var Mildly variable (Cannon and Stobie 1973; Lloyd-Evans 1977a). $\sigma_{m}(V-K) \approx 0.1 \mathrm{mag}$.

No $U-B$ available. $V$ and $B-V$ from Bessell and Norris 1976.

No $U-B$ available. $V$ and $B-V$ from ROA catalog.

$U B V$ data from Geyer 1967.

Highly variable. $(V-K)_{0}$ predicted from $(J-K)_{0}$ - see text. $\sigma_{m}(V-K) \approx 0.2 \mathrm{mag}$. $(B-V)_{0}$ is $(\langle B\rangle-\langle V\rangle)_{0}$ from ROA catalog.

5 Somewhat variable. $(V-K)_{0}$ is an average of $(\langle V\rangle-K)_{0}$ and $(V-K)_{0}$ predicted from $(J-K)_{0}-$ see text. $\sigma_{m}(V-K) \approx$ 0.15 mag. $(B-V)_{0}$ is $(\langle B\rangle-\langle V\rangle)_{0}$ from ROA catalog.

6 Infrared measurements made on JD 2,443,699.

7 Infrared measurements made on JD 2,443,705.

ROA 320, the variations in $V$ are substantial, and $(V-K)_{0}$ values were derived from the observed $(J-K)_{0}$ values by using a plot of $(V-K)_{0}$ against $(J-K)_{0}$ for all the other $\omega$ Cen stars. (The $\left[(J-K)_{0}\right.$, $\left.(V-K)_{0}\right]$-plot used for this purpose is Fig. 7 of this paper and is discussed in the Appendix. For V6, the mean relationship was linearly extrapolated to obtain $\left.(V-K)_{0}.\right)$ We cannot rule out the possibility that for these large-amplitude variables our derived values of $(V-K)_{0}$ are off by several tenths of a magnitude. Simultaneous measurements at $V$ and $K$ are necessary to study the behavior of the colors of these stars. For the remaining variables, the excursions in $V$ are not so large, and two values of $(V-K)_{0}$ were found. First, $(V-K)_{0}$ was found from $(J-K)_{0}$ as for V6,
V17, and ROA 320; second, the mean $V$ value from Dickens $e t$ al. was used with our single $K$ observation to give a value of $(V-K)_{0}$. Consideration of the dispersion in $V$ values for these stars shows that an average of the two values should give a $(V-K)_{0}$ accurate to $\sim 0.15 \mathrm{mag}$. The uncertainties finally adopted are noted in Table 1 .

\section{RESULTS}

\section{a) A Wide Range in $\mathrm{CO}$ Absorption}

The first of the main results of this paper is that at a given $(V-K)_{0}$ color, the spread in $\mathrm{CO}$ index among the $\omega$ Cen giants is several times greater than that which could be accounted for by observational 


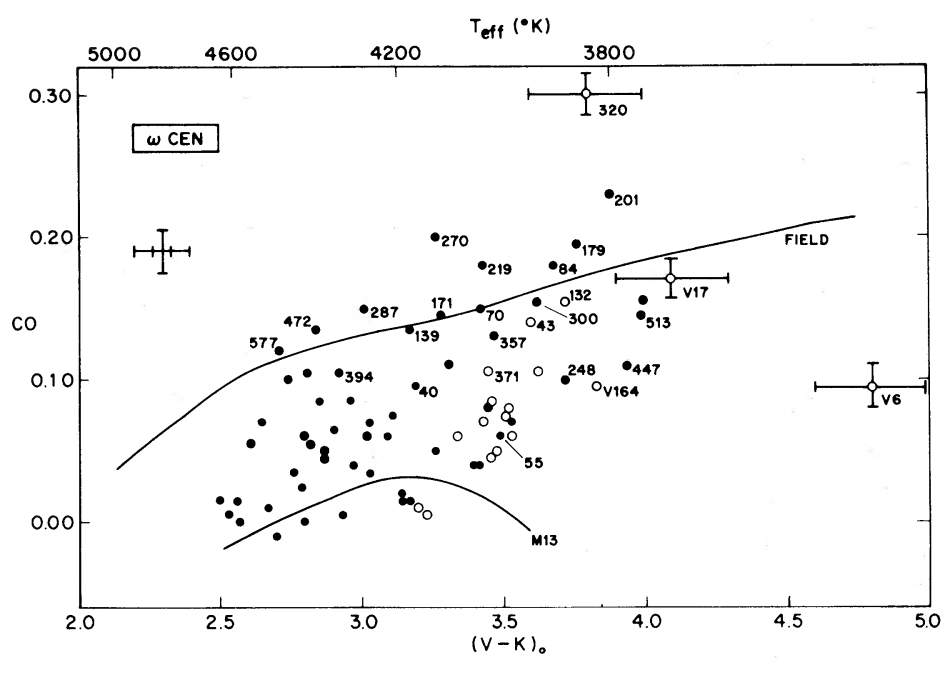

FIG. $1 a$

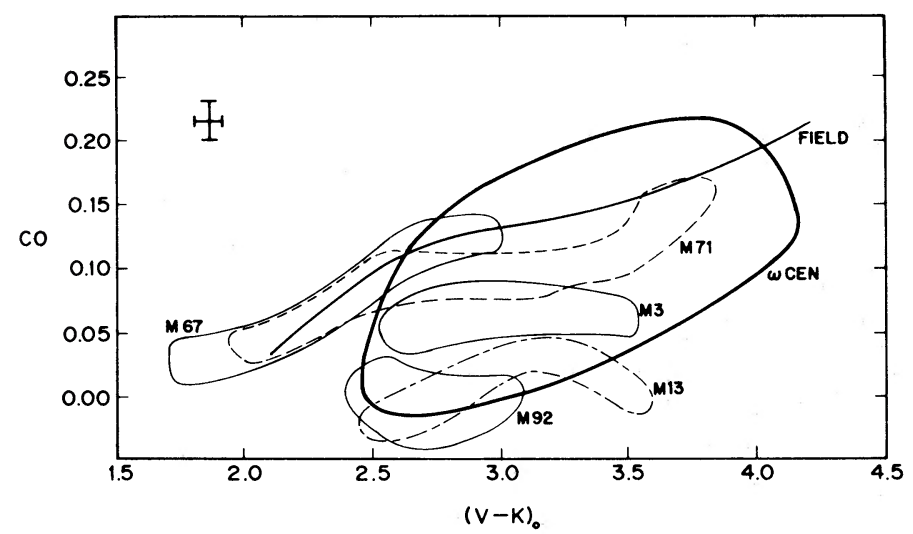

FIG $1 b$

FIG. 1.-(a) The CO index is plotted as a function of $(V-K)_{0}$ color (corrected for reddening), with some of the spectroscopically notable stars labeled (see Table 1). The $T_{\text {eff }}$ scale was derived from the atmospheric models given in CFP, as discussed in the text. The filled circles are nonvariables, and the open circles are variables. For the variables, the longer of the two $(V-K)_{0}$ error bars in the upper left-hand corner applies. The shorter error bar applies to all the nonvariables. The larger uncertainties for V6, V17, and ROA 320 are discussed in the text. The M13 and field fiducial lines are from CFP and Frogel $e t$ al. (1978). The dispersions of the individual $\mathrm{CO}$ values about these lines for stars in clusters other than $\omega$ Cen is approximately equal to the nominal error bar. This can also be judged from Fig. $1 b$. (b) $\mathrm{CO}$ index versus $(V-K)_{0}$ for the envelopes of the distributions of the individual stars in several clusters (CFP; Frogel, Persson, and Cohen 1979). The envelopes enclose all of the observed stars in M92, M13, M3, and M67, but do not enclose stars B and 29 in M71, or V6, V17, and ROA 320 in $\omega$ Cen. The single error bar is representative of most of the data points.

uncertainties alone. This is illustrated in Figure $1 a$. The spread in CO index for $\omega$ Cen is also considerably greater than that determined for other globular clusters. Figure $1 b$ shows the envelope of the distribution for $\omega$ Cen compared with those for clusters studied previously (CFP; Frogel, Persson, and Cohen 1979). (These envelopes exclude two stars in $\omega$ Cen and in M71.) For these comparison clusters the spread in $\mathrm{CO}$ index at a given $(V-K)_{0}$ is consistent with the observational uncertainties. The distribution of $\mathrm{CO}$ indices among the $\omega$ Cen stars extends from M92like stars with slightly negative $\mathrm{CO}$ indices to the region of solar abundance stars with $\mathrm{CO}$ indices near $0.20 \mathrm{mag}$, and the variation in $\mathrm{CO}$ is smooth in that at a given $(V-K)_{0}$ there is neither any bifurcation in the distribution nor any tendency for the stars to cluster at a preferred value of $\mathrm{CO}$ index.

\section{b) The Width of the Giant Branch in $(V-K)_{0}$}

The giant branch of $\omega$ Cen is significantly wider in $(V-K)_{0}$ than either the measurement errors or the corresponding branches for other clusters. This result is evident from Figure 2, where the $K_{0}$ magnitudes of the $\omega$ Cen stars are plotted against the $(V-K)_{0}$ colors. This behavior is apparent at all luminosities for which we have data - a range of $4 \mathrm{mag}$ in $K_{0}$. Also shown are the giant-branch loci for M92, M13, and M71, drawn using the data of CFP and Frogel, Persson, and Cohen (1979). A further result is that 


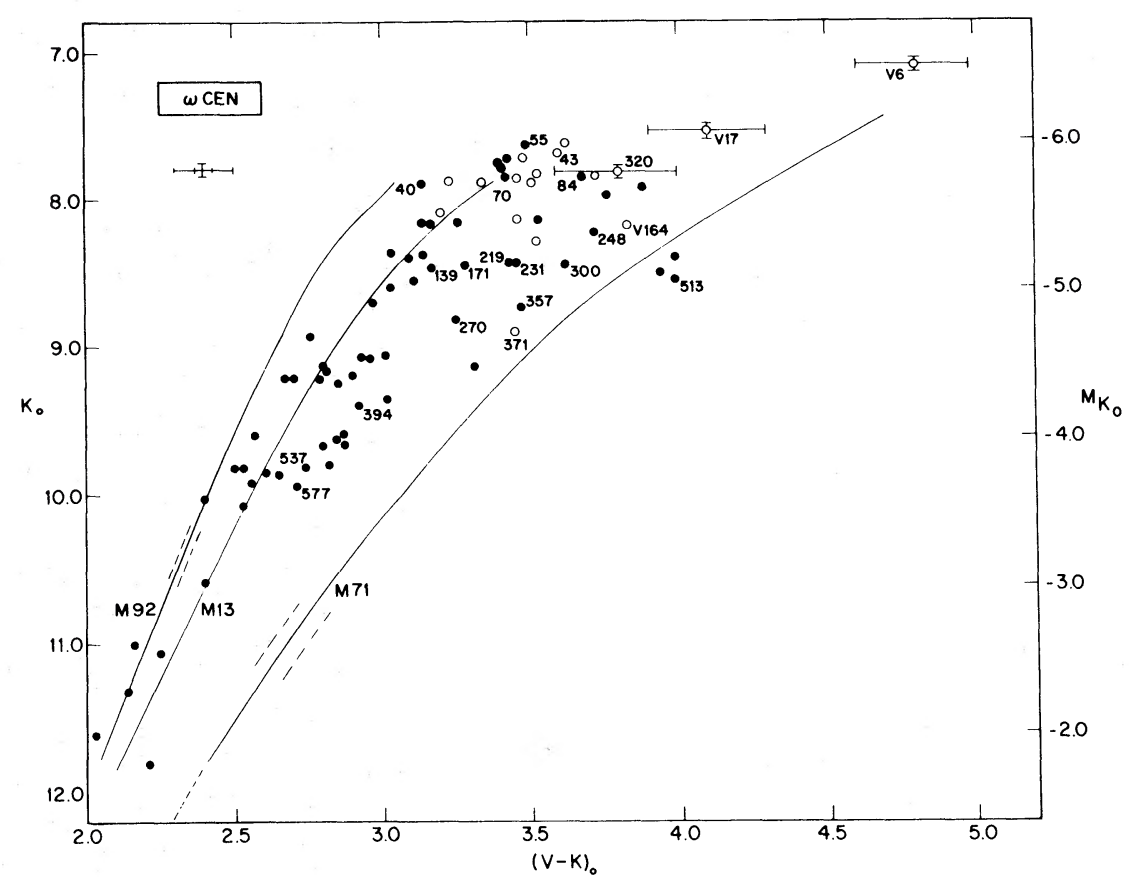

Fig. 2. $-K_{0}$ versus $(V-K)_{0}$ for red giants in $\omega$ Cen. As in Fig. $1 a$, the nominal error bar applies to the nonvariables [ $f$ filled circles, shorter $(V-K)_{0}$ error bar] and the variables [open circles, longer $(V-K)_{0}$ error bar], except V6, V17, and ROA 320 . The fiducial lines for the three other clusters are from CFP and Frogel, Persson, and Cohen (1979). The representative $1 \sigma$ dispersion about these lines is noted by the short dashed lines on the M71 and M92 loci. (The dispersion for M13 equals that for M92.) It is approximately independent of luminosity. The reddening corrected distance moduli assumed to locate the fiducial lines (i.e., the $M_{K_{0}}$ axis) are 13.60, 14.57, 14.33, and 12.90 mag for $\omega$ Cen, M92, M13, and M71, respectively (Harris 1976; CFP; Frogel, Persson, and Cohen 1979).

only a few of the $\omega$ Cen stars lie near the M71 locus, in contrast to the appearance of Figure $1 a$, where many of the stars have $\mathrm{CO}$ indices which exceed those of the M71 stars at a given $(V-K)_{0}$.

In CFP it was shown that $(V-K)_{0}$ can be calibrated accurately in terms of effective temperature to give a value of $T_{\text {eff }}$ which is quite insensitive to $[\mathrm{Fe} / \mathrm{H}]$ or surface gravity for metal poor stars. The spread in $(V-K)_{0}$ at a given luminosity is not likely to arise from differential blanketing or back-warming effects (see n. 6 in $\S \mathrm{Va}$ below). We therefore attribute the spread in $(V-K)_{0}$ among the $\omega$ Cen stars at a given luminosity to a spread in $T_{\text {eff }}$. The location in $T_{\text {eff }}$ of the giant branch at a given luminosity depends mostly on metal abundance (Hoyle and Schwarzschild 1955; Sandage and Smith 1966), and more recently Renzini (1977) and Rood (1978) have shown that it is only the heavy-metal abundance (i.e., $\mathrm{Fe}$ and not $\mathrm{CNO}$ ) that is important. Age cannot have a significant effect because the range in age required to produce a spread in $(V-K)_{0}$ of $0.7 \mathrm{mag}$ at a given luminosity is in excess of $10^{10}$ years, as calculated from the analytical expressions of Renzini (1977). Thus we are led to conclude that the spread in $(V-K)_{0}$ in the $\left[K_{0},(V-K)_{0}\right]-$ diagram is due mostly to a spread in heavy metals among the stars in $\omega$ Cen. This result is not new; rather, it corroborates recent results on the $[\mathrm{Fe} / \mathrm{H}]$ and $[\mathrm{Ca} / \mathrm{H}]$ abundances among the RR Lyrae stars (Butler, Dickens, and Epps 1978; Freeman and Rod- gers 1975, 1978) and the giants (Cohen 1978; Norris 1978).

\section{THE SPREAD IN CO INDEX AND COLOR ON THE UPPER GIANT BRANCH}

The question we shall now consider is how the spread in $\mathrm{CO}$ absorption compares with the spread in $(V-K)_{0}$ color among the $\omega$ Cen giants and what this tells us about the abundances of the CNO elements relative to the heavy elements. The $\mathrm{CO}$ data should be capable of providing new information on this question because $\mathrm{CO}$ formation controls the formation of other carbon molecules as long as $\mathrm{C}<\mathrm{O}$. We shall form an empirical calibration of $\mathrm{CO}$ index versus metal abundance and effective temperature based on observations of stars in other globular clusters.

\section{a) The Spread in $\mathrm{CO}$}

In order to characterize the spread in $\mathrm{CO}$, we first define for each star a $\mathrm{CO}$ strength relative to a suitable lower-bound fiducial line. The M92 and M13 stars, which one could in principle choose to take as the lower bound of the $\mathrm{CO}$ distribution, do not extend to colors sufficiently red to establish the lower bound beyond $(V-K)_{0}=3.5$; furthermore, we do not know how strong the $\mathrm{CO}$ bands actually are in these cluster stars. We thus use as a lower bound $\mathrm{CO}$ indices which would be measured in a (fictitious) zero metal 
abundance star. A good approximation to this is made by defining the lower bound at a given $(V-K)_{0}$ to be the $\mathrm{CO}$ index which would be measured for a blackbody at a $T_{\text {eff }}$ corresponding to $(V-K)_{0}$. Because the CO index is defined to be zero for $\alpha$ Lyr $\left(T_{\text {eff }} \approx 10,000 \mathrm{~K}\right)$, the lower-bound locus is slightly negative for all temperatures less than this.

By simply taking the effective magnitudes of a blackbody at 2.20 and $2.36 \mu \mathrm{m}$, we define the lowerbound locus $\mathrm{CO}_{\mathrm{bb}}$ to be

$$
\begin{aligned}
& \mathrm{CO}_{\mathrm{bb}}=-0.0029-0.0135(V-K)_{0} \\
& \text { for } 4.5>(V-K)_{0}>1.3 .
\end{aligned}
$$

Because the CO index is also a function of $T_{\text {eff }}$ at a given $[\mathrm{Fe} / \mathrm{H}]$, as shown by the field and M71 loci in Figure $1 b$, we remove the temperature dependence to first order by normalizing the $\mathrm{CO}$ strengths to those expected for a solar metal abundance star. The mean field locus of $\mathrm{CO}$ versus $(V-K)_{0}$ is suitable for this purpose, and we define this locus to be $\mathrm{CO}_{\odot}$. Figure $3 a$ illustrates the normalization procedure. The adopted definition of $\mathrm{CO}$ absorption relative to that expected for solar composition is then

$$
R(\mathrm{CO}) \equiv \frac{\mathrm{CO}(\text { index })-\mathrm{CO}_{\mathrm{bb}}}{\mathrm{CO}_{\odot}-\mathrm{CO}_{\mathrm{bb}}}
$$

Table 2 lists the values of $R(\mathrm{CO})$ for the $\omega$ Cen stars. The effect on $R(\mathrm{CO})$ of adjusting the $\mathrm{CO}$ indices by the function $\mathrm{CO}_{\mathrm{bb}}$ (which amounts to 0.05 mag at $4000 \mathrm{~K}$ ) is very small.

\section{b) The Spread in $(V-K)_{0}$}

We seek to convert the observed spread in $(V-K)_{0}$ in $\omega$ Cen into an estimate of the heavy-metal abundance relative to the Sun for each star. These estimates, denoted as $R(V-K)$ values, are based on the progressions of giant-branch loci redward with increasing

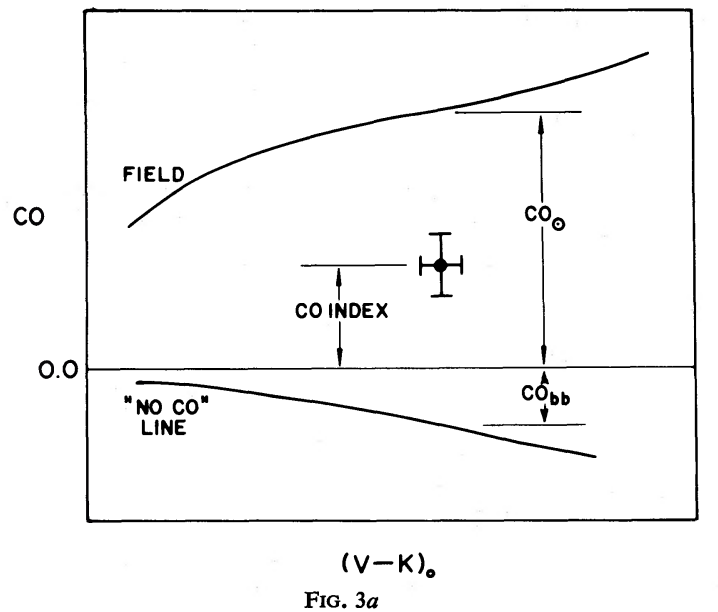

$[\mathrm{Fe} / \mathrm{H}]$. The theoretical result that the giant-branch location in $T_{\text {eff }}$ is controlled mostly by $[\mathrm{Fe} / \mathrm{H}]$ and not $[\mathrm{CNO} / \mathrm{H}]$ is assumed to be valid. We adopt the following scheme, illustrated in Figure $3 b$. We use the two best defined sequences available at high and low metal abundance, namely, those of M92 and M71 (CFP; Frogel, Persson, and Cohen 1979), and calculate for each star the quantity

$\left[(V-K)_{0}-(V-K)_{\mathrm{M} 92}\right] /\left[(V-K)_{\mathrm{M} 71}-(V-K)_{\mathrm{M} 92}\right]$, where $(V-K)_{\mathrm{M} 92}$ and $(V-K)_{\mathrm{M} 71}$ are the colors of the M92 and M71 ridge lines at the same value of $M_{K_{0}}$. The colors are understood to be corrected for reddening. ${ }^{4}$ This definition normalizes the shift in $(V-K)_{0}$ from the M92 line to the total shift between M92 and M71. However, we want to normalize to a solar metal abundance, in analogy with the definition of $R(\mathrm{CO})$. The only suitable available giant-branch locus is that of M67, which, however, does not extend brighter than $M_{K_{0}}=-3.0$ because of the sparseness of the cluster. At this $M_{K_{0}}$, the difference in $(V-K)_{0}$ between the M92 and M71 loci is 0.71 as large as that between M92 and M67. Thus, to normalize to a solar metal abundance, we multiply the shifts relative to M71 and M92 by 0.71 . Thus

$R(V-K)=\frac{(V-K)_{0}-(V-K)_{\mathrm{M} 92}}{(V-K)_{\mathrm{M} 71}-(V-K)_{\mathrm{M} 92}} \times 0.71$.

Table 2 lists the values of $R(V-K)$ calculated from equation (3).

The set of $R(V-K)$ values can provide only a crude ranking of the $\omega$ Cen stars in heavy-metal abundance, and spectroscopic determinations of the abundances of the individual giants are naturally to be preferred. Nevertheless, the $R(V-K)$ values have the

${ }^{4}$ For values of $M_{K 0}$ brighter than the M92 tip, a small extrapolation of the M92 sequence was assumed.

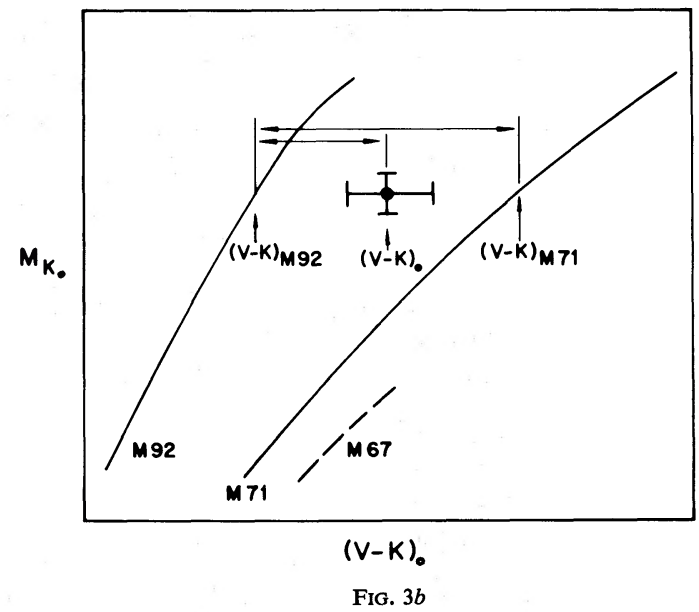

Fig. 3.- (a) Schematic CO versus $(V-K)_{0}$ plot showing the parameters used to define $R(\mathrm{CO})$. See $\S \mathrm{IV} a$. (b) Schematic colormagnitude diagram showing the parameters used to define $R(V-K)$. See $\S \mathrm{IV} b$. The long and short horizontal arrows depict the denominator and numerator of the first factor of eq. (3). 


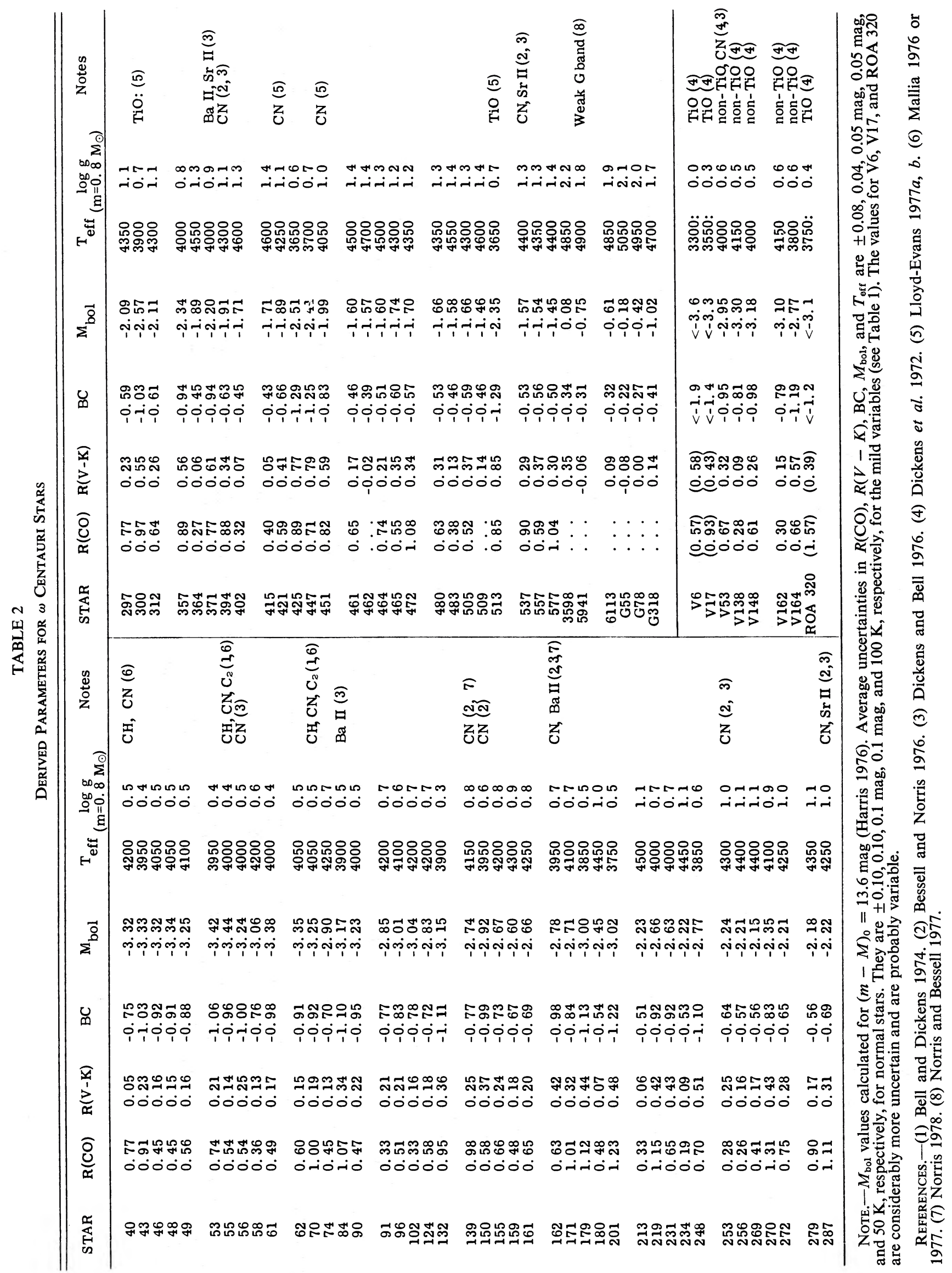




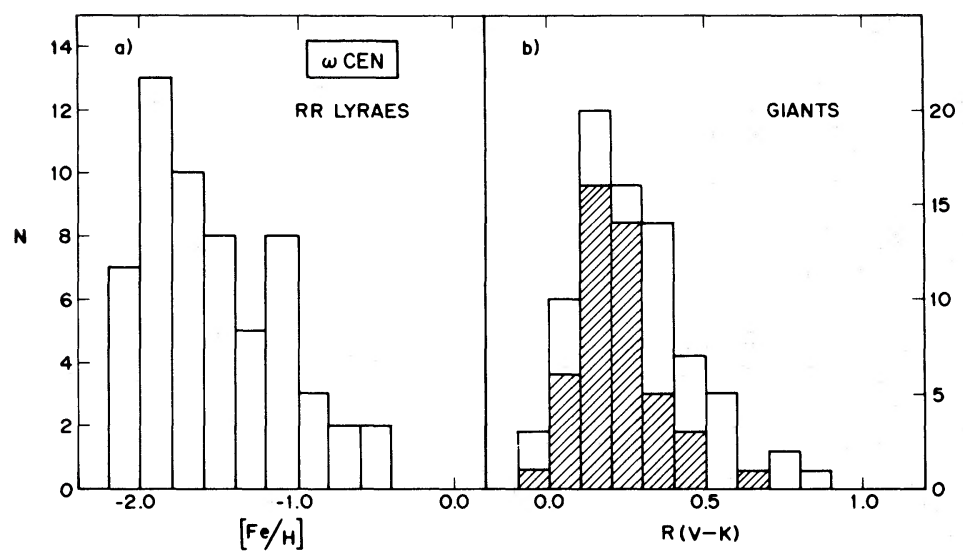

FIG. 4.- (a) Histogram of the distribution over metal abundance, in bins 0.2 wide in $[\mathrm{Fe} / \mathrm{H}]$, for 58 RR Lyrae variables from Butler et al. (b) Histogram of the distribution over $R(V-K)$ for the data of this paper. The hatched area represents only those stars in Cannon and Stobie's (1973) photometrically unbiased sample.

advantage of being purely empirical and can be compared directly to $R(V-K)$ values computed in the same way for all the stars in several calibrating clusters. This is discussed in $\S \mathrm{IV} c$.

An indirect check on the procedure is provided by looking at the distribution over $[\mathrm{Fe} / \mathrm{H}]$ of the $\mathrm{RR}$ Lyrae stars studied by Butler et al. A histogram of the 58 stars in their Table 4 is shown in Figure 4, together with the distribution over $R(V-K)$ for our 82 stars in Table 2. We have not attempted to transform our $R(V-K)$ values to $[\mathrm{Fe} / \mathrm{H}]$ estimates. The two distributions differ in detail but share a basic similarity in having a large fraction of the stars at a low value of metal abundance, near -1.7 , and a long tail of stars extending to near solar abundance.

\section{c) Comparison of the Spread in $\mathrm{CO}$ with that in $(V-K)_{0}$}

Figure 5 presents the dependence of $R(\mathrm{CO})$ on $R(V-K)$. To avoid crowding, the figure has been plotted twice so that stars showing enhanced $\mathrm{CN}, \mathrm{CH}$, $s$-process elements, and $\mathrm{TiO}$ can be picked out and so that the individual stars can be easily identified. The

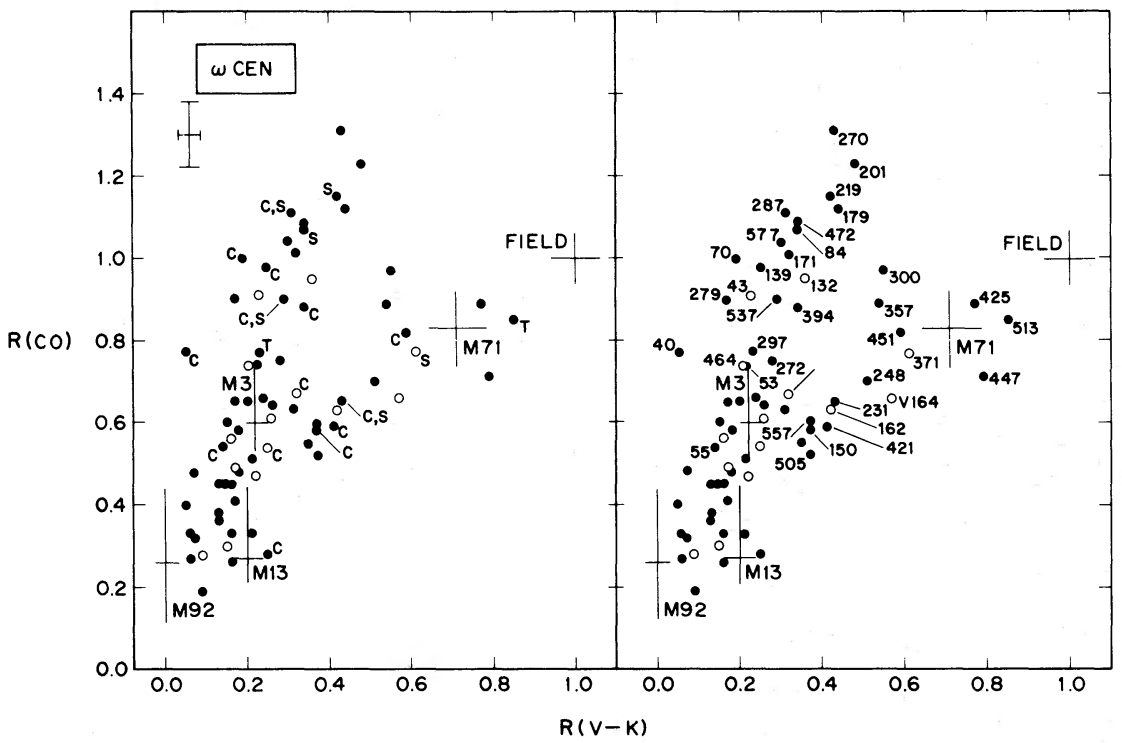

FIG. 5.-The parameter $R(\mathrm{CO})$ versus metallicity parameter $R(V-K)$ for the $\omega$ Cen giants, excluding V6, V17, and ROA 320, for which the $R(V-K)$ values are uncertain by $\sim 0.5$. The same distribution is plotted twice: in the left panel the $\mathrm{CN}, \mathrm{C}_{2}$, and $\mathrm{CH}$ stars (C), the $s$-process stars (S), and the TiO stars (T) are identified, and in the right panel the individual ROA or variable numbers are given. Open circles are variables, and filled circles are nonvariables. The single "error" bar in the upper left-hand corner shows the average uncertainty based on the observational errors but does not include uncertainties arising from the locations of the fiducial lines required to define $R(\mathrm{CO})$ and $R(V-K)$. The crosses representing M92, M13, M3, M71, and the field (and M67) stars are centered on the median $R(\mathrm{CO})$ and $R(V-K)$ values for all the stars in each cluster, and the arms of the crosses extend to the quartiles of these distributions. 
$R(\mathrm{CO})$ and $R(V-K)$ values for the calibrating clusters are shown in Figure 5 as crosses which locate the medians of the distribution of $R(\mathrm{CO})$ with $R(V-K)$ among the individual cluster stars. The arms of the crosses extend to the quartiles of the distributions. The sizes of these regions measure the spread in $R(\mathrm{CO})$ and $R(V-K)$ within the calibrating clusters; this spread arises from a combination of measurement uncertainties, possible real differences among the stars in each cluster, and our implicit assumptions regarding the similarity of the $\mathrm{CO}$ and $V-K$ loci in going from cluster to cluster. The single error bar in the upper left of Figure 5 represents a typical error bar for each of the $\omega$ Cen stars.

From the spread in $\mathrm{CO}$ and $V-K$ in Figures 1 and 2 , we would expect a range or spread in the $[R(\mathrm{CO})$, $R(V-K)$ ]-plot of Figure 5. Rather than a smooth relationship, however, we see a distinct tendency for the stars to separate into two groups. A majority of the stars display $\mathrm{CO}$ absorption which is strong for their $R(V-K)$ values, while the remainder follow a steady increase of $R(\mathrm{CO})$ with $R(V-K)$ which follows the M92-M13-M71 sequence. The "CO strong" stars in Figure 5 also show an increase of $\mathrm{CO}$ absorption with $R(V-K)$.

The simplest conclusion we can draw is that many of the upper-giant-branch stars in $\omega$ Cen have anomalously high $\mathrm{CO}$ absorption for their metal abundance. ${ }^{5}$

We can find no strong evidence for any other systematic photometric differences between the two groups of stars in various two-color diagrams (discussed in the Appendix) except possibly in a plot of $(B-V)_{0}$ versus $(V-K)_{0}$. In this diagram the $\mathrm{CO}$ weak stars appear to be on average 0.04 mag bluer in $(B-V)_{0}$ at a given $(V-K)_{0}$ than the $\mathrm{CO}$ strong stars. We do not consider this difference to be significant.

\section{d) $\mathrm{CO}$ and $[\mathrm{Fe} / \mathrm{H}]$ Variations}

What is the explanation for the distribution of points in Figure 5 ? Is it necessary to invoke primordial variations in $[\mathrm{CNO} / \mathrm{Fe}]$ as well as $[\mathrm{Fe} / \mathrm{H}]$, or are mixing processes alone sufficient? Norris (1978) has suggested that primordial variations in $\mathrm{N}$ and $s$-process element abundances relative to $\mathrm{Fe}$ may be required to account

${ }^{5} \mathrm{~A}$ possible problem with any interpretation of Fig. 5 is that asymptotic branch stars from the metal rich side of the giant branch are contaminating the sample of (supposedly) metal poor, $\mathrm{CO}$ strong stars. This is not likely to be the case for several reasons. First, there are more $\mathrm{CO}$ strong stars than normal stars, which is reversed from the empirical ratio of asymptotic giant-branch stars to giant-branch stars in other clusters. Second, because the distribution over heavy-metal abundance is both smooth and strongly weighted to the metal poor domain, the total fraction of $\mathrm{CO}$ strong stars that are actually metal rich asymptotic giant-branch stars must be small, and no bifurcation in the $[R(\mathrm{CO}), R(V-K)]$-distribution in Fig. 5 would be expected. An additional argument is that many asymptotic giant-branch stars in metal poor clusters are weak-G-band stars (Norris and Zinn 1977) and that these should have, if anything, slightly weakened $\mathrm{CO}$ absorption in analogy with weak-G-band field stars (Hartoog, Persson, and Aaronson 1977). for his observed correlation of $\mathrm{Ca}$ and $\mathrm{CN}$ index variations among a sample of $\omega$ Cen giants. A problem with the interpretation of both the present results and those of Norris (1978) is the incomplete empirical calibration of the $\omega$ Cen measurements against both "standard" globular clusters and bright field giants and the lack of theoretical spectral synthesis of the relevant molecular bands. Nevertheless, the purely empirical evidence of Figure 5 indicates that there must be a mechanism operating in addition to $[\mathrm{Fe} / \mathrm{H}]$ variations; abundance variations with all metals varying together would produce only a single sequence in the $[R(\mathrm{CO}), R(V-K)]$-diagram. The evidence relating to $[\mathrm{CNO} / \mathrm{Fe}]$ variations, mixing of $\mathrm{CN}$ and $\mathrm{CNO}$ processed material, or mixing of triple- $\alpha$ material as being the necessary additional process operating in $\omega$ Cen does not provide a clear-cut solution to the problem, as discussed below.

We first note that the difference in mean $\mathrm{CO}$ strength between M3 and M13, two clusters of similar [Fe/H] (CFP; Pilachowski 1978), is evident in Figure 5 . The relative locations of the M3 and M13 stars lead us to speculate that a common mechanism is operating to produce both the M3-M13 CO anomaly and the spread in $\mathrm{CO}$ within $\omega \mathrm{Cen}$. This hypothesis requires the giants in M3 together with the $\omega$ Cen stars on the more vertical "CO strong" sequence in Figure 5 to have been subject to some process in their past history different from that to which the M13 giants and the $\omega$ Cen stars along the more horizontal "CO weak" sequence have been subject.

Available spectroscopic data argue against the possibility that primordial variations in $[\mathrm{CNO} / \mathrm{Fe}]$ produce the two sequences. If this process were dominant, one might expect all the CO strong stars to have strong $\mathrm{CN}$ and $\mathrm{CH}$ bands, but this is not the case. Although there are $\mathrm{CN}$ strong and $\mathrm{CH}$ strong stars among the $\mathrm{CO}$ strong stars in Figure 5 (see references in Table 2), not all the $\mathrm{CO}$ strong stars are $\mathrm{CN}$ strong and some of the $\mathrm{CO}$ weak stars are $\mathrm{CN}$ strong. Furthermore, the existence of a bifurcation rather than a spread in $R(\mathrm{CO})$ value for a given $R(V-K)$ (and therefore for $[\mathrm{Fe} / \mathrm{H}]$ ) does not seem likely, as any nucleosynthetic process which synthesizes just the light elements should create a more continuous range in $[\mathrm{C} / \mathrm{Fe}]$ or $[\mathrm{CO} / \mathrm{Fe}]$.

The hypothesis that mixing controls the distribution of stars in Figure 5 also has some inconsistencies. We note that the $s$-process stars appear in both branches of Figure 5. The production and mixing of $s$-processed material to the surface thus appears to be independent of whatever process leads to the $[\mathrm{C}$ or $\mathrm{O} / \mathrm{Fe}]$ variations. Better statistics on the numbers of $s$-process stars as a function of metal abundance and $\mathrm{CO}$ strength would be most valuable.

If one considers the possibility that mixing produced the $\mathrm{CO}$ strong sequence from (assumed) normal $[\mathrm{CNO} / \mathrm{Fe}]$ stars on the $\mathrm{CO}$ weak sequence (which coincides with the M92-M13-M71-field sequence), another inconsistency appears. Neither $\mathrm{CN}$ cycle nor equilibrium CNO cycle processing plus mixing can produce stars with both $\mathrm{CN}$ and $\mathrm{CO}$ enhancements, as 
in each case the total atmospheric carbon abundance and hence the $\mathrm{CO}$ column density will decrease. Thus, to produce the $\mathrm{CO}$ strong sequence from giants along the $\mathrm{CO}$ weak sequence, mixing of fresh triple- $\alpha$ carbon to the surface must occur. The established membership of three bona fide carbon stars in $\omega$ Cen also implies triple- $\alpha$ mixing or large primordial variations in [CNO/Fe].

An alternative mixing scenario is that the $\mathrm{CO}$ weak sequence is produced from the $\mathrm{CO}$ strong sequence by $\mathrm{CN}$ cycle and/or equilibrium $\mathrm{CNO}$ cycle processing plus mixing. In this picture carbon has been severely depleted in the stars lying along the $\mathrm{CO}$ weak sequence, and the relative absence of stars between the two groups of Figure 5 implies that the $\mathrm{C}$ depletion occurs rapidly after the onset of mixing. Such $\mathrm{CN}$ cycle mixing with resulting $\mathrm{C}$ depletion and $\mathrm{N}$ enhancement has been inferred for the more luminous giants in M92 and NGC 6397 (Carbon, Kraft, and Langer 1979; Bell, Dickens, and Gustafsson 1978), while the lowergiant-branch stars in these same clusters have normal $[\mathrm{CNO} / \mathrm{Fe}]$. The effects of $\mathrm{CN}$ cycle mixing have also been observed in stars as hot as $T_{\text {eff }} \approx 4500 \mathrm{~K}$ (corresponding to $\left.M_{\text {bol }} \approx+0.5\right)$, as is shown by ${ }^{12} \mathrm{C} /{ }^{13} \mathrm{C}$ ratios near 20 in substantial numbers of field red giants (Tomkin, Luck, and Lambert 1976) and by direct spectroscopic analysis of a limited number of stars (Lambert and Ries 1977).

If the $\mathrm{C}$ depletion in normal globular cluster giants as a function of luminosity follows that of the field giants, then the appearance of the M71, M13, and M92 stars as a single point for each cluster in Figure 5 can be maintained. We then hypothesize that in M3 (where $\mathrm{CO}$ photometry for only the more luminous giants is available [CFP]) and in some of the $\omega$ Cen giants, the $\mathrm{C}$ depletion expected for stars near the red giant tip has not occurred. It is possible that the lack of mixing in most or all of the M3 stars and in some of the $\omega$ Cen stars can be connected with the distribution of core rotational velocity in these red giants, if mixing occurs via meridional circulation, as suggested by Sweigart and Mengel (1979).

We made an attempt to reproduce theoretically the principal features in Figure 5 by using the formalism in Frogel, Persson, and Cohen (1979) to describe the variation in $\mathrm{CO}$ strength as a function of metallicity and by using the Ciardullo-Demarque tracks (Ciardullo and Demarque 1977) calibrated with the observations of CFP to determine $R(V-K)$ as a function of $\mathrm{Fe} / \mathrm{H}$. A C depletion of approximately -0.8 dex for stars on the $\mathrm{CO}$ weak sequence compared to stars on the $\mathrm{CO}$ strong sequence will reproduce the observed relative slopes of the sequences in Figure 5. This is close to the deduced $\mathrm{C}$ depletion produced by $\mathrm{CN}$ cycle mixing as observed in other globular clusters (Carbon, Kraft, and Langer 1979; Bell, Dickens, and Gustafsson 1978).

This scenario, attractive though it may seem, also has several problems. If we believe that the $\mathrm{CO}$ weak sequence represents those stars which have undergone $\mathrm{CN}$ cycle mixing, then only a minority of the more luminous red giants in $\omega$ Cen have mixed. Further- more, both theoretical calculations (Sweigart and Mengel 1979) and an analysis of stars in M92 (Bell, Dickens, and Gustafsson 1978) show that the stars furthest down the giant branch are most likely to be unmixed. Yet of the 14 stars in Figure 5 with $M_{\text {bol }}>$ -2.0 , three probably lie along the $\mathrm{CO}$ weak sequence, which we have ascribed to stars which have already undergone $\mathrm{CN}$ cycle mixing.

We therefore see that there is no clear indication of the identity of the mechanism, operating in addition to variations in $[\mathrm{Fe} / \mathrm{H}]$, which controls the distribution of points in an $[R(\mathrm{CO}), R(V-K)]$-plot for the $\omega$ Cen giants. Most likely, a complicated combination of possible primordial variations in $[\mathrm{CNO} / \mathrm{Fe}]$ and mixing to the surface of $\mathrm{CN}$ cycle material as well as material enhanced in fresh (triple- $\alpha$ ) carbon will ultimately be required to explain the entire range of phenomena seen in the $\omega$ Cen giants.

\section{e) The Second Parameter}

We now consider the identification of the "second parameter" in light of our new results on $\omega$ Cen. The possibility that $[\mathrm{CNO} / \mathrm{Fe}]$ could be the second parameter that controls horizontal-branch morphology in globular clusters was suggested by Hartwick and McClure (1972) and discussed recently by Castellani and Tornambe (1977). The M3-M13 CO anomaly also led Pilachowski (1978) and CFP to tentatively identify $[\mathrm{CNO} / \mathrm{Fe}]$ as the second parameter because M3 contains numerous red horizontal-branch stars and M13 has none. By this argument, $\omega$ Cen should also contain numerous red horizontal-branch stars since a majority of its giants resemble those in M3 in having relatively strong $\mathrm{CO}$ absorption for their metal abundance (Fig. 5). The ordinary color-magnitude diagram of those $\omega$ Cen stars having a high probability of membership shows, however, that the cluster contains few, if any, red horizontal-branch stars (Woolley et al. 1966). If the anomalies in CO ranking among different clusters and within $\omega$ Cen arise from primordial variations in $[\mathrm{CNO} / \mathrm{Fe}]$, then the present results contradict the hypothesis that $[\mathrm{CNO} / \mathrm{Fe}]$ is the second parameter.

On the other hand, if these anomalies arise from mixing, then it seems likely that simple ranking schemes based on one or another molecular abundance $(\mathrm{CN}$, $\mathrm{CH}, \mathrm{CO}$ ) could be misleading in attempts to ascertain whether $[\mathrm{CNO} / \mathrm{Fe}]$ is the second parameter. In this case the physical parameters controlling mixing (e.g., internal rotation in the case of meridional circulation) and their uniformity from star to star and from cluster to cluster must be understood first.

\section{f) Spatial Distributions}

The wide distribution of metal abundance for the giants in $\omega$ Cen is analogous to that found in galaxies. Is there also a radial gradient of metal abundance 
within $\omega$ Cen as there is in galaxies? Plots of $R(\mathrm{CO})$ and $R(V-K)$ versus radial position in the cluster do not show strong effects. There is a weak tendency for the very reddest stars in $(V-K)_{0}$ to lie closer to the cluster center, consistent with the idea of a radial abundance gradient in the normal sense, but a similar plot of $[\mathrm{Fe} / \mathrm{H}]$ for the $58 \mathrm{RR}$ Lyrae variables studied by Butler et al. shows no such effect. There is no discernible dependence of $R(\mathrm{CO})$ on radial distance. These results are consistent with the integrated-light $U B V$ data of Chun and Freeman (1979), which show no radial color gradients in the outer parts of the cluster. Further work on the possible existence of a radial gradient in the abundance distribution would be of interest, as differences in the distributions of various element tracers could give us direct evidence on the sequence(s) of metal enrichment in the protocluster gas cloud.

\section{PHYSICAL PARAMETERS}

\section{a) Luminosities and Temperatures}

Bolometric corrections (BC) and bolometric luminosities, found by integrating the observed energy distributions from $U$ to $K$ as described in CFP, are listed in Table 2. Small extrapolations were used to correct for flux missing shortward of $3600 \AA$ and longward of $2.4 \mu \mathrm{m}$.

The absolute calibration is that of Wilson et al. (1972), and the BC scale is normalized so that $\mathrm{BC}($ Sun $)=-0.08$ (Allen 1963). The simple integration scheme does not explicitly take into account sources of opacity that are present in the $\omega$ Cen stars but not implicitly included in the absolute calibration used. Nevertheless, an integration over the energy distribution of the Sun, using only the broad-band colors in Allen (1963) gives the correct solar bolometric luminosity. [The procedure adopted here is slightly different from that used in CFP, where BC(Sun) was taken to be 0.0.] For the variables V6, V17, and ROA 320 , it is likely that the luminosities are underestimated, and the $M_{\text {bol }}$ values in Table 2 are given as lower limits. Glass and Feast (1973) obtained a $K-L$ color of $\sim 0.4 \mathrm{mag}$ for $\mathrm{V} 6$, and this large value could indicate that a substantial fraction of the energy is coming out longward of $2 \mu \mathrm{m}$. Further photometry at longer wavelengths is required to check on this.

Values of $T_{\text {eff }}$, determined using the values of $(V-K)_{0}$ given in Table 1 (corrected for CO absorption as discussed in CFP) and the CFP calibration, are listed in Table 2 . In computing the values of $T_{\text {eff }}$, we took account of variations in $\log g$ among the stars. (Effects due to $[\mathrm{Fe} / \mathrm{H}]$ variations are smaller and were ignored.) First, trial values of $T_{\text {eff }}$ were found from the $\log g=1.0,[\mathrm{Fe} / \mathrm{H}]=-1.5$ sequence of models in CFP. Values of $\log g$ (Table 2) were then found by using the values of $M_{\mathrm{bor}}$ listed in Table 2 and by assuming that $\mathfrak{M}=0.8 \mathfrak{M}_{\odot}$ for all the stars. Finally, small corrections to $T_{\text {eff }}$ were made to account for the

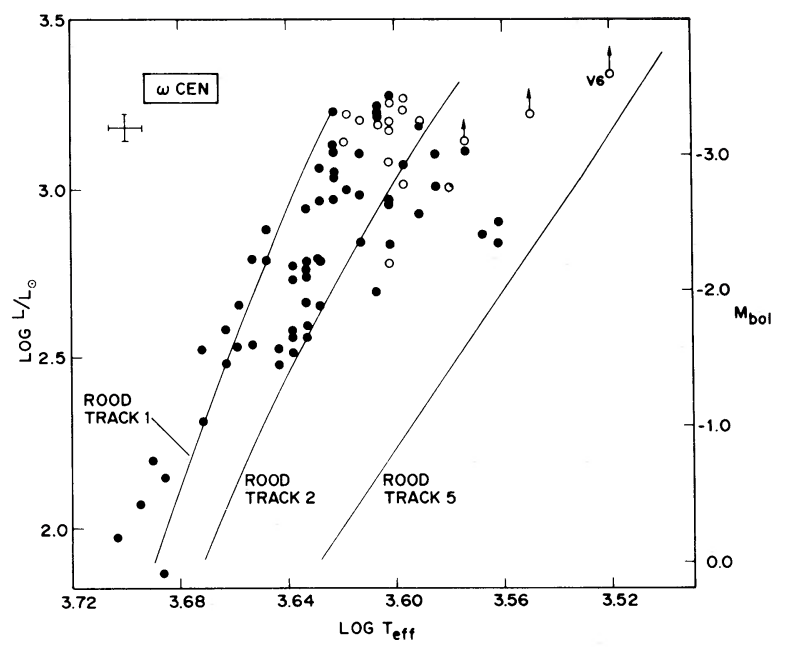

Fig. 6. -The H-R diagram in physical units for $\omega$ Cen giants. The computation of the bolometric luminosities and effective temperatures is described in the text. The single error bar represents most of the data points and includes uncertainties arising from the model atmosphere $T_{\text {eff }}$ calibration and from observational errors. Uncertainties in the distance modulus are not included. The three theoretical evolutionary tracks from Rood (1972) correspond to $\mathfrak{M}=0.8 \mathfrak{M}_{\odot}, X=0.7$, $Y=0.3$, and $Z / Z_{\odot}=0.005$ (track 1), $Z / Z_{0}=0.05$ (track 2$)$, and $Z / Z_{\odot}=0.5$ (track 5).

difference between the calculated and assumed values of $\log g$. Typical adjustments were no more than $30 \mathrm{~K}^{6}{ }^{6}$

Figure 6 shows the relation between $M_{\text {bol }}$ and $T_{\text {eff }}$ for the $\omega$ Cen stars. This plot is entirely analogous to the infrared color-magnitude diagram of Figure 2, and we include it here only for the sake of completeness. The features noted in Figure 2 are reproduced: the spread in $T_{\text {eff }}$ resulting from a spread in $[\mathrm{Fe} / \mathrm{H}]$ is apparent, and there is good agreement of the luminosity of the giant branch tip for $\omega$ Cen compared to the theoretical tracks of Rood (1972). It was noted in CFP that the slopes of the cluster giant branches are flatter near the tip than are the theoretical tracks. This is also a feature of the $\omega$ Cen distribution.

The variable V6 is apparently somewhat brighter than the tip, a fact first noted by Glass and Feast

${ }^{6}$ For the coolest stars there are several effects which could give rise to inaccurate temperatures. First, strong $\mathrm{CN}$ bands cause back warming of the atmosphere and thus an overestimate of $T_{\text {eff }}$. Several of the stars displaying very red $(V-K)_{0}$ colors have strong $\mathrm{CN}$ bands. From the calculations of Gustafsson et al. (1975) we estimate that this effect is not likely to exceed $\sim 100 \mathrm{~K}$, corresponding to a change in $V-K$ of $0.16 \mathrm{mag}$ at $(V-K)_{0}=3.2$. Second, blanketing effects could be serious in the peculiar stars. This was checked directly, using new echelle spectra of eight stars. For ROA 55, which is probably the most strongly blanketed star in the cluster, the total flux removed by lines from the continuum within the bandpass of the $V$ filter was $29 \%$ compared to $8 \%$ for ROA 58 , a star of comparable luminosity on the blue edge of the giant branch. This difference of $\sim 0.2 \mathrm{mag}$ in $V$ is not very large - if there were no compensating blocking in the $2.2 \mu \mathrm{m}$ band, it would correspond to a temperature error of $120 \mathrm{~K}$. Nevertheless, high-resolution spectra at $2 \mu \mathrm{m}$ and model atmospheres which include molecular opacities will be required to refine the temperatures for the metal rich and peculiar stars. 
(1973). V6 is probably an asymptotic giant-branch star, and higher-spectral-resolution observations of it would be most interesting.

\section{b) Comparison with $T_{\text {eff }}$ Determined from $R-I$ Photometry}

The values of $T_{\text {eff }}$ in Table 2 can be compared with published values derived from $R-I$ photometry. Dickens and Bell (1976) have tabulated temperatures for a number of $\omega$ Cen giants derived from $R-I$ and $B-V$ colors, via the Gustafsson et al. (1975) models. There are eight stars in common for which existing photometry yields "direct" temperatures, and 19 in common for which "indirect" temperatures were found from $B-V$ (see Dickens and Bell's discussion and their Table 2).

The temperature comparison is given in Table 3, which lists, in successive columns, the average of the differences, the dispersion, and the number of stars in common. The comparison shows that the $V-K$ temperatures average somewhat more than $100 \mathrm{~K}$ hotter than temperatures from $R-I$ photometry. This corresponds to only 0.04 mag in $R-I$ color but $0.25 \mathrm{mag}$ in $(V-K)_{0}$ at $4000 \mathrm{~K}$. The difference could reflect a problem in our calibration, but we note an internal disagreement between the $R-I$ and $B-V$ temperatures listed by Dickens and Bell (1976) which amounts to $85 \mathrm{~K}$ for the sample of 27 stars. In fact, the dispersion in the differences between the $R-I$ and $B-V$ temperatures is rather large $(150 \mathrm{~K})$. Because of this dispersion, small differences in the atmospheric models used, and the probable effects of unaccounted for opacity sources at $V, R, I$, and $K$, we feel that the $R-I$ and $V-K$ temperatures are in reasonable agreement.

\section{c) Comparison with DDO Photometry}

Observations of $\omega$ Cen giants on the DDO system have been published by Bessell and Norris (1976), Norris and Bessell (1975), and Hesser, Hartwick, and McClure (1976, 1977). Bessell and Norris (1976) have calculated $T_{\text {eff }}$ values for a sample which includes 10 stars in common with us, of which five were found spectroscopically to have strong $\mathrm{CN}$ bands (see Table 2). Several of the stars in this sample, however, have DDO colors which do not lie within the range of Osborn's (1973) $T_{\text {eff }}$ calibration.

TABLE 3

TEMPERATURE COMPARISON ${ }^{a}$

\begin{tabular}{ccrr}
\hline Comparison & Average & \multicolumn{1}{c}{$\sigma$} & $N$ \\
\hline$T(V-K)-T(R-I)_{\text {direct } \ldots \ldots \ldots} \ldots+150$ & 100 & 8 \\
$T(V-K)-T(R-I)_{\text {indirect } \ldots \ldots \ldots}+100$ & 140 & 19 \\
$T(V-K)-T(B-V) \ldots \ldots \ldots$ & +25 & 155 & 27 \\
$T(R-I)-T(B-V) \ldots \ldots \ldots$ & -85 & 150 & 27 \\
$T(V-K)-T(\mathrm{DDO})_{\mathrm{CN} \text { strong } \ldots \ldots \ldots} \ldots \ldots+380$ & 85 & 5 \\
$T(V-K)-T(\mathrm{DDO})_{\mathrm{CN} \text { weak } \ldots \ldots} \ldots+35$ & 125 & 5
\end{tabular}

${ }^{a} T(V-K)$ from Table 2 of this paper. $T(B-V)$ and $T(R-I)$ from Dickens and Bell 1976. See text for explanation.
The comparison of the DDO and $V-K$ temperatures is given in Table 3 . The strong $\mathrm{CN}$ stars average nearly $400 \mathrm{~K}$ cooler in $T(\mathrm{DDO})$ than in $T(V-K)$. Inspection of Bessell and Norris's (1976) Table 1 shows that the stars which fall outside the DDO calibration (e.g., ROA 70) have very large $C(41-42)$ and $C(42-45)$ colors, normally indicative of cool temperatures. It seems plausible that the $4000 \AA$ opacity source discovered by Bond and Neff (1969) and discussed by Bessell and Norris (1976) as a possible contributor to the spread in $B-V$ can significantly affect the DDO colors. The 41,42 , and 45 filters of the DDO system lie near the edge of the $4000 \AA$ absorption feature, and a simple computation shows that the rather low values of $T$ (DDO) compared to either $T(V-K)$ or $T(R-I)$ can be explained by a modest absorption over this wavelength region.

Comparison of the DDO $\mathrm{CN}$ index with the $\mathrm{CO}$ indices in Table 1 for the 16 stars in common with us shows no obvious correlation. As pointed out by Hesser, Hartwick, and McClure (1977), the CN index is not uniquely related to temperature-sensitive indices, particularly for stars near the red giant tip. In view of the possible contamination of the DDO CN and temperature indices by the Bond-Neff opacity, it seems that derivation of estimates for the amount of excess $\mathrm{CN}$ absorption from DDO photometry for giants in $\omega$ Cen may be quite difficult. Spectroscopic observations of violet $\mathrm{CN}$ strengths, discussed recently by Norris (1978), should give much better values for the degree of $\mathrm{CN}$ enhancement, and direct comparison with the $\mathrm{CO}$ indices should be quite interesting.

\section{SUMMARY}

The main results of this paper can be summarized as follows.

1. The infrared photometry presented here for 82 stars in $\omega$ Cen demonstrates that at a fixed luminosity, the giant branch can be as wide as 0.8 mag in $(V-K)_{0}$. This can best be explained by a spread of heavyelement abundances among the stars that is in qualitative agreement with that obtained from spectroscopic observations of RR Lyrae variables and red giants. This spread in $[\mathrm{Fe} / \mathrm{H}]$ is about a factor of 30 .

2. Narrow-band $\mathrm{CO}$ measurements have been presented here for 74 members of $\omega$ Cen, and the $\mathrm{CO}$ indices show a wide variation from star to star at constant $(V-K)_{0}$ color which has a form quite different from the distribution in $(V-K)_{0}$, hence in $[\mathrm{Fe} / \mathrm{H}]$, at constant $K_{0}$. Although the distribution in $(V-K)_{0}$ is strongly peaked toward blue colors equivalent to low metallicities, the distribution of the $\mathrm{CO}$ indices is such that some stars lie near the empirical relationship for solar metallicity field giants. By comparing the spread in $\mathrm{CO}$ indices with that in $(V-K)_{0}$ (Fig. 5), we find two groups of stars, one with significantly larger $\mathrm{CO}$ indices for their $T_{\text {eff }}$ and metallicity than the other. A majority of the stars belong to this latter group. Explanations involving primordial variations in $\mathrm{C} / \mathrm{Fe}$ and mixing were discussed, with evidence presented for and against each. It seems likely that 
both effects are operating and that this segregation is related to the M3-M13 anomaly.

We are grateful to M. Clark, G. Forrester, A. Guerra, L. Papic, and F. Peralta for their help with the equipment and observations at Las Campanas and to
J. Baldwin and J. Rios for their help at CTIO. We thank J. Baldwin, J. Elias, B. Lasker, G. Neugebauer, F. Schweizer, and R. Zinn for discussions, and $\mathbf{P}$. Gilman, N. Newton, and J. Bedke for help in preparing the manuscript. This work was supported in part by NSF grant 76-22676.

\section{APPENDIX \\ TWO-COLOR DIAGRAMS FOR $\omega$ CENTAURI GIANTS}

In this Appendix we present several two-color diagrams which illustrate the behavior of the ordinary and spectroscopically peculiar $\omega$ Cen giants compared to stars in other globular clusters.

$$
\text { I. THE }\left[(J-K)_{0},(V-K)_{0}\right] \text {-DIAGRAM }
$$

Figure 7 shows the dependence of $(J-K)_{0}$ on $(V-K)_{0}$. None of the variables at the end of Table 1 with a 4 or 5 in the "notes" column appear on the plot, as it is this correlation that was used in $\S$ II $c$ to estimate $(V-K)_{0}$ for these stars from their $(J-K)_{0}$ values. The "mean line" on Figure 7 is the relationship actually used for this purpose. The observed spread in the data is consistent with the spread expected from observational uncertainties.

The dashed line shows the mean relationship established from observations of 96 stars, spread out evenly between $(V-K)_{0}=2.0$ and 3.9, in M3, M13, M92, M67, M71, and M5 (CFP; Frogel, Persson, and Cohen 1979; unpublished data). There are no systematic differences among the relationships for the different clusters, and the dispersion about the line is no greater than 0.03 mag. Johnson's (1966) mean $J-K$ and $V-K$ colors for field giants also lie close to the dashed line after transformation to our photometric system via the equation $[J-K]$ $($ here $)=0.92[J-K]($ Johnson $)+0.03$ (Frogel et al. 1978). The mean line through the $\omega$ Cen points and the dashed line are not coincident. However, until details of the transformation between the $\omega$ Cen observations and those of CFP are better known, it is not certain that the small shift is real.

$$
\text { II. THE }\left[(J-H)_{0},(H-K)_{0}\right] \text {-DIAGRAM }
$$

The $\left[(J-H)_{0},(H-K)_{0}\right]$ two-color diagram is shown in Figure 8. The distribution of most of the stars in the diagram is qualitatively similar to the composite distribution of stars in M3, M13, M92, M71, M5, and M67 in two respects. First, there is a rather slow dependence of $(H-K)_{0}$ on $(J-H)_{0}$, and second, the reddest nonvariable stars (ROA 300, ROA 513) do not display the turnover in $(J-H)_{0}$ near $(J-H)_{0}=0.80$ that characterizes the colors of field giants.

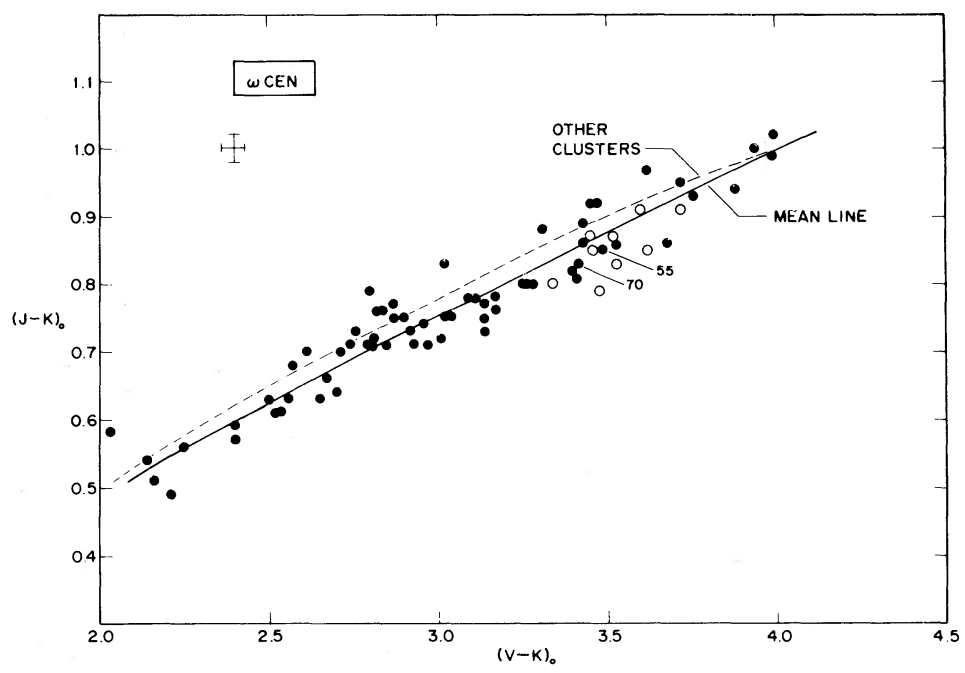

Fig. 7.- $(J-K)_{0}$ versus $(V-K)_{0}$ for $\omega$ Cen giants. Filled circles, nonvariables; open circles, mild variables. The mean line through the $\omega$ Cen points is an eye fit to the data. It was used to derive estimates of $(V-K)_{0}$ for the small-amplitude variables (see Table 1) and also for V6, V17, and ROA 320. An extrapolation was required for V6. The dashed line is the ridge line drawn through the 96 stars of the clusters studied previously (see text). The offset between the two ridge lines is discussed in the text. 


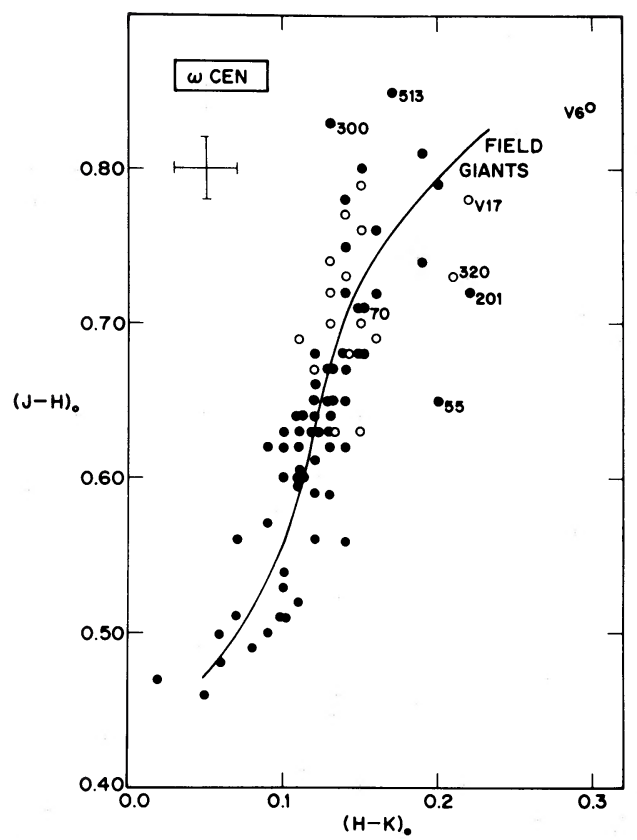

FIG. 8.-The $\left[(J-H)_{0},(H-K)_{0}\right]$-diagram for $\omega$ Cen (open circles, variables; filled circles, nonvariables) compared to the relationship for field giants from Frogel et al. (1978). The single error bar refers to all the points and also characterizes the dispersion for field giants. Except for V6, V17, ROA 55, ROA 320, and ROA 201, the stars lie close to those in M3 and M13 (CFP).

Five stars stand well apart from the rest in Figure 8 in having $(H-K)_{0}$ colors apparently too red for their $(J-H)_{0}$ colors. These are the large-amplitude variables V6, V17, and ROA 320, the carbon star ROA 55, and ROA 201. The colors of the three variables resemble those of late-type variables in the field.

ROA 55, which is not a variable, displays a clear excess $(H-K)_{0}$ color; its $J H K$ colors are similar to those of warm carbon stars in the LMC (work in preparation). Interestingly, the milder $\omega$ Cen carbon star in our sample, ROA 70, does not show this effect. In view of the strong $\mathrm{CH}, \mathrm{CN}$, and $\mathrm{C}_{2}$ bands seen in the visible in ROA 55, it seems possible that bands arising from these and other molecules are affecting the broad-band infrared colors at the $0.1 \mathrm{mag}$ level. Note also that the CO index of ROA 55 is 0.09 mag less than that of ROA 70 . Alternatively, there could be excess emission at $2 \mu \mathrm{m}$ arising from a dust shell.

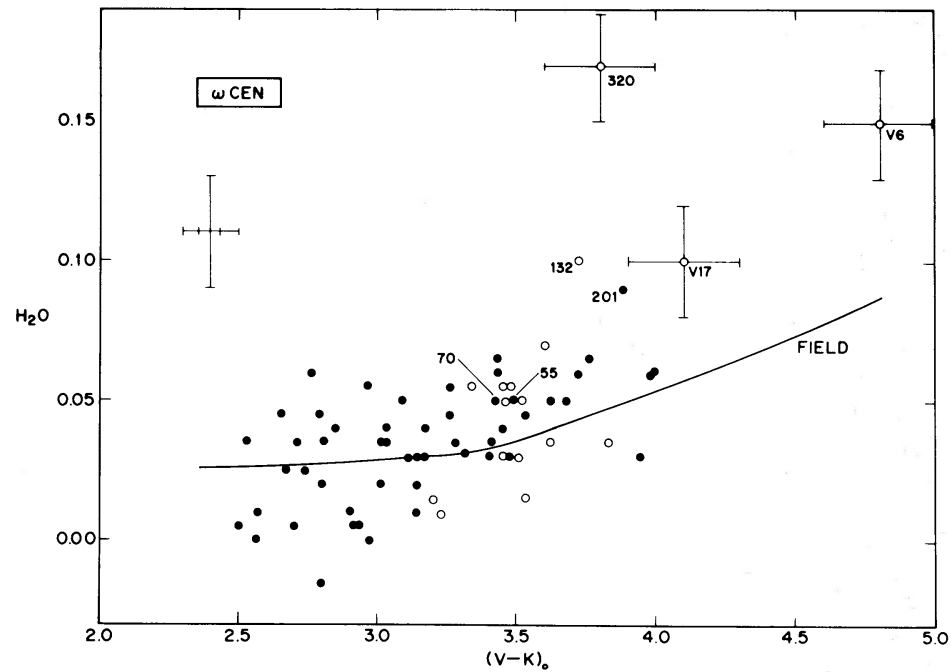

Fig. 9.- The $\mathrm{H}_{2} \mathrm{O}$ index versus $(V-K)_{0}$. Open circles, variables, longer $(V-K)_{0}$ error bar; filled circles, nonvariables, shorter $(V-K)_{0}$ error bar. The positive $\mathrm{H}_{2} \mathrm{O}$ index of the field line, from Aaronson et al., does not indicate the presence of $\mathrm{H}_{2} \mathrm{O}$ absorption at this temperature, merely the slope of the stellar continuum. The locations of V6, V17, and ROA 320 are discussed in the text. 


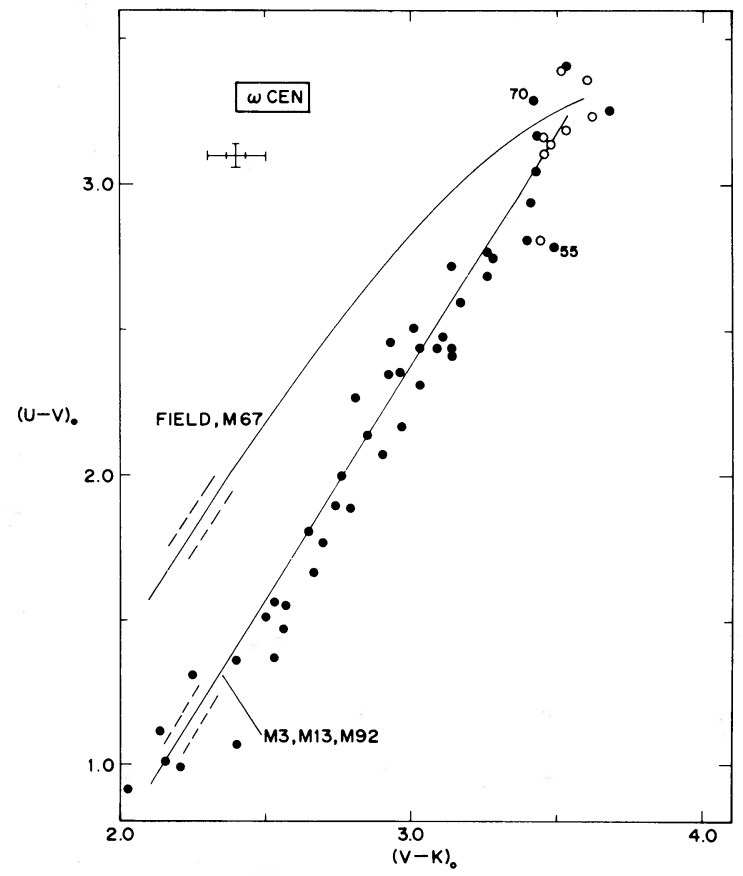

FIG. 10. $-(U-V)_{0}$ versus $(V-K)_{0}$ for $\omega$ Cen giants compared to ridge lines for other clusters (CFP) and field stars (Frogel et al. 1978). The two carbon stars ROA 55 and ROA 70 are marked. Open circles, variables, longer error bar; filled circles, nonvariables, shorter error bar. Several of the variables in Table 1 and in other figures cannot be included because of a lack of data. The two dashed lines associated with the two loci show the approximate $\pm 1 \sigma$ dispersion of the data points for these sequences. The dispersion is independent of color.

\section{THE $\mathrm{H}_{2} \mathrm{O}$ INDICES}

Figure 9 shows the distribution of the $\mathrm{H}_{2} \mathrm{O}$ indices of the $\omega$ Cen stars. The scatter in the $\mathrm{H}_{2} \mathrm{O}$ indices is generally consistent with the measurement errors, and the ridge line of the main group of stars having $\mathrm{H}_{2} \mathrm{O}$ indices $<0.07$ mag is well reproduced by the mean relation for field giants. Neither the $\omega$ Cen stars nor the field stars with $\mathrm{H}_{2} \mathrm{O}$ $<0.07 \mathrm{mag}$ at this effective temperature actually have detectable $\mathrm{H}_{2} \mathrm{O}$ absorption. Rather, the slightly positive indices are produced simply because the energy distributions are red compared to that of $\alpha$ Lyr, which is defined to have an $\mathrm{H}_{2} \mathrm{O}$ index of 0.0 .

For ROA 320, the combination of strong $\mathrm{H}_{2} \mathrm{O}$ and strong $\mathrm{CO}$ absorption (see Fig. $1 a$ ) is similar to that seen in ordinary Mira variables or giants near spectral type M5. The $(J-K)_{0}$ color of ROA 320 at the time of observation, however, does not correspond to an M5 III star, but rather to about M0 III; stars of this color display $\mathrm{H}_{2} \mathrm{O}$ and $\mathrm{CO}$ absorption far weaker than is observed in ROA 320.

$$
\text { IV. THE }\left[(U-V)_{0},(V-K)_{0}\right] \text {-DIAGRAM }
$$

Figure 10 shows a plot of the $(U-V)_{0}$ colors against the $(V-K)_{0}$ colors. The $\omega$ Cen giants lie close to the mean relationship for M3, M13, and M92, and although the scatter for the $\omega$ Cen stars is slightly larger than expected from the measurement errors, it is consistent with the scatter in the distribution for M3, M13, and M92 taken together and consistent with the idea that a heavy-metal abundance range exists within the cluster. It is interesting that the two carbon stars ROA 55 and ROA 70 have similar values of $(V-K)_{0}$ but quite different values of $(U-V)_{0}$.

$$
\text { v. THE }\left[(B-V)_{0},(V-K)_{0}\right] \text {-DIAGRAM }
$$

It is important to directly compare these colors because it was on the basis of the ordinary $(V, B-V)$ colormagnitude diagram that the idea of a spread in heavy metals within $\omega$ Cen was introduced, and it is this spread that we claim determines the appearance of the $\left[K_{0},(V-K)_{0}\right]$-plot. The data, plotted in Figure 11 , shows a tight correlation. The scatter for $(V-K)_{0}<3.5$ is consistent with that for the metal poor clusters taken together; the smooth line through the $\omega$ Cen distribution is the ridge line for the metal poor clusters. The agreement shows that for most of the stars excess opacity effects at $B$, as discussed by Bessell and Norris (1976), do not exceed $0.1 \mathrm{mag}$ on average, provided, of course, that $(V-K)_{0}$ is, as we claim, primarily sensitive to $T_{\text {eff }}$ and not affected by blanketing as well. The other smooth curve is the ridge line through the M67 and field giants, which agrees with 


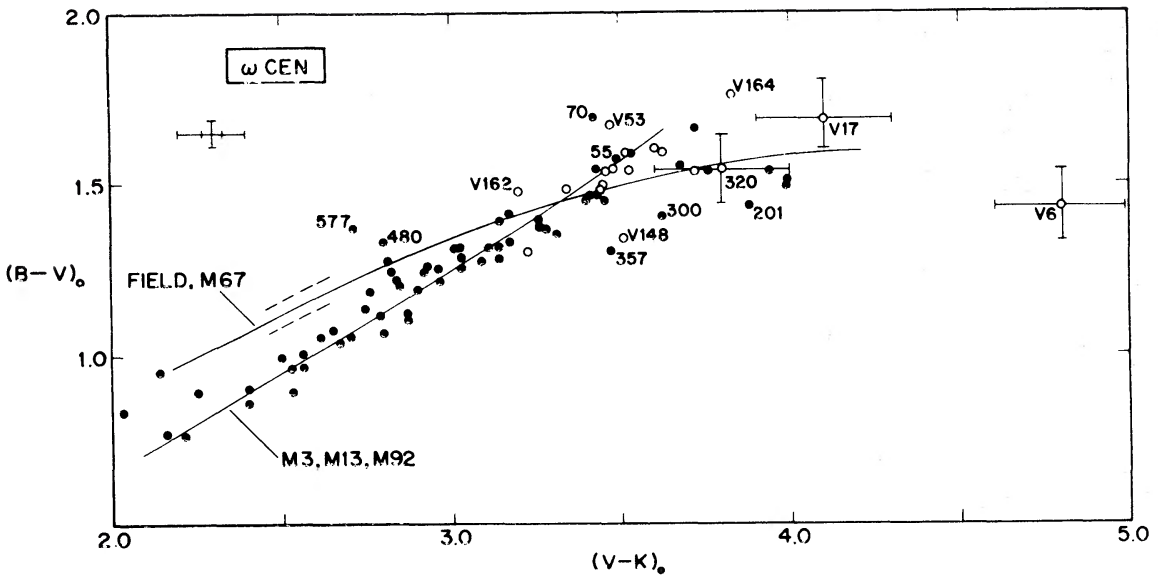

Fig. 11.- $(B-V)_{0}$ versus $(V-K)_{0}$ for $\omega$ Cen giants. Open circles, variables, longer $(V-K)_{0}$ error bar; filled circles, nonvariables, shorter $(V-K)_{0}$ error bar. Dashed lines show the $\pm 1 \sigma$ dispersion for the data of both comparison sequences.

Johnson's (1966) mean relationship. The two lines cross over at $(V-K)_{0} \approx 3.5 \mathrm{mag}$, and as is well known, $B-V$ loses most of its sensitivity to effective temperature for oxygen rich stars redder than this. Beyond $(V-K)_{0}$ $=3.5 \mathrm{mag}$, the variables are well spread out in $(V-K)_{0}$ but not in $(B-V)_{0}$.

\section{REFERENCES}

Aaronson, M., Frogel, J. A., and Persson, S. E. 1978, Ap. J., $220,442$.

Allen, C. W. 1963, Astrophysical Quantities (London: Athlone Press).

Bell, R. A., and Dickens, R. J. 1974, M.N.R.A.S., 166, 89.

Bell, R. A., Dickens, R. J., and Gustafsson, B. 1978, in Astronomical Papers Dedicated to Bengt Strömgren, ed. A. Reiz and T. Andersen (Copenhagen: Copenhagen University Observatory), p. 249.

Bessell, M. S., and Norris, J. 1976, Ap. J., 208, 369; 210, 618.

Bond, H. E. 1975, Ap. J. (Letters), 202, L47.

Bond, H. E., and Neff, J. S. 1969, Ap. J., 158, 1235.

Butler, D., Dickens, R. J., and Epps, E. 1978, Ap. J., 225, 148.

Cannon, R. D., and Stobie, R. S. 1973, M.N.R.A.S., 162, 207.

Carbon, D. F., Kraft, R. P., and Langer, G. E. 1979, preprint.

Castellani, V., and Tornambe, A. 1977, Astr. Ap., 61, 427.

Chun, M. S., and Freeman, K. C. 1979, Ap. J., 227, 93.

Ciardullo, R. B., and Demaraue, P. 1977, Trans. Astr. Obs. Yale Univ., Vol. 35.

Cohen, J. G. 1978, presentation at NATO Advanced Study Institute on Globular Clusters.

Cohen, J. G., Frogel, J. A., and Persson, S. E. 1978, Ap. J., 222, 165 (CFP).

Dickens, R. J. 1972, M.N.R.A.S., 159, 7P.

Dickens, R. J., and Bell, R. A. 1976, Ap. J., 207, 506.

Dickens, R. J., Feast, M. W., and Lloyd-Evans, T. 1972 , M.N.R.A.S., 159, 337.

Dickens, R. J., and Woolley, R. v. d. R. 1967, Roy. Obs. Bull., No. 128.

Feast, M. W. 1973, in IAU Colloquium No. 21, Variable Stars in Globular Clusters and in Related Systems, ed. J. D. Fernie (Dordrecht: Reidel), p. 131.

Freeman, K. C., and Rodgers, A. W. 1975, Ap. J. (Letters), 201, L71.

1978, presentation at NATO Advanced Study Institute on Globular Clusters.

Frogel, J. A., Persson, S. E., Aaronson, M., and Matthews, K. 1978, Ap. J., 220, 75.

Frogel, J. A., Persson, S. E., and Cohen, J. G. 1979, Ap. J., 227, 499.

Geyer, E. H. 1967, Zs. Ap., 66, 16.

Glass, I. S., and Feast, M. W. 1973a, M.N.R.A.S., 163, 245; 164, 424.

1977, M.N.R.A.S., 181, 509

Gustafsson, B., Bell, R. A., Eriksson, K., and Nordlund, $\AA$. 1975, Astr. Ap., 42, 407.
Harding, G. A. 1962, Observatory, 82, 205.

Harris, W. E. 1976, A.J., 81, 1095.

Hartoog, M. R., Persson, S. E., and Aaronson, M. 1977, Pub. A.S.P., 89, 660 .

Hartwick, F. D. A., and McClure, R. D. 1972, Ap. J. (Letters), 176, L57.

Hesser, J. E., Hartwick, F. D. A., and McClure, R. D. 1976, Ap. J. (Letters), 207, L113.

. 1977, Ap.J. Suppl., 33, 471.

Hoyle, F., and Schwarzschild, M. 1955, Ap. J. Suppl., $2,1$.

Johnson, H. L. 1966, Ann. Rev. Astr. Ap., 4, 193.

Lambert, D. L., and Ries, L. M. 1977, Ap. J., 217, 508.

Lloyd-Evans, T. 1977a, M.N.R.A.S., 178, 345 1977b, M.N.R.A.S., 181, 591 .

1978, presentation at NATO Advanced Study Institute on Globular Clusters.

Mallia, E. A. 1976, Astr. Ap., 48, 129.

. 1977, Astr. Ap., 60, 195.

Martin, W. C. 1938, Leiden Ann., Vol. 17, No. 2.

Norris, J. 1978, presentation at NATO Advanced Study Institute on Globular Clusters.

Norris, J., and Bessell, M. S. 1975, Ap. J. (Letters), 201, L75.

. 1977, Ap. J. (Letters), 211, L91.

Norris, J., and Zinn, R. 1977, Ap. J., 215, 74.

Osborn, W. 1973, Ap. J., 186, 725.

Persson, S. E., and Frogel, J. A. 1978, presentation at NATO Advanced Study Institute on Globular Clusters.

Pilachowski, C. A. 1978, Ap. J., 224, 412.

Renzini, A. 1977, in Advanced Stages in Stellar Evolution, ed. P. Bouvier and A. Maeder (Geneva: Geneva Observatory), p. 149.

Rood, R. T. 1972, Ap. J., 177, 681.

. 1978, presentation at NATO Advanced Study Institute on Globular Clusters.

Sandage, A., and Smith, L. L. 1966, $A p . J ., 144,886$.

Sweigart, A. V., and Mengel, J. G. 1979, Ap. J., 229, 624.

Tomkin, J., Luck, R. E., and Lambert, D. L. 1976, Ap. J., 210, 694.

Wilson, W. J., Schwartz, P. R., Neugebauer, G., Harvey, P. M., and Becklin, E. E. 1972, Ap. J., 177, 523.

Wing, R. F. 1973, in IAU Colloquium No. 21, Variable Stars in Globular Clusters and in Related Systems, ed. J. D. Fernie (Dordrecht: Reidel), p. 164

Wing, R. F., and Stock, J. 1973, Ap. J., 186, 979

Woolley, R. v. d. R., et al. 1966, Roy. Obs. Ann., No. 2. 
Note added in proof.-The effective temperatures in this paper were derived from $(V-K)_{0}$ from the models of CFP with an interpolation to the Johnson (1966) scale for stars cooler than the model grid. Recently Ridgway, Joyce, White, and Wing (1980, Ap. J., in press) have presented a fundamental recalibration of the effective temperature scale for cool giants. The effect of the new $T_{\text {eff }}$ scale upon the results of this paper is to raise the effective temperatures in Table 2 for stars cooler than $4000 \mathrm{~K}$. It is not clear that a straight application of the new scale to derive better $T_{\text {eff }}$ values from $(V-K)_{0}$ will be correct because the new scale applies strictly only to solar metal abundance stars having some TiO absorption at $V$. Nevertheless, a general increase in $T_{\text {eff }}$ of order $100 \mathrm{~K}$ for a tabulated value on the old scale of $3750 \mathrm{~K}$ is indicated. None of the conclusions of this paper is affected by this change. Figure 6 will be altered slightly, and it now appears that the flattening of the giant branch loci near the tip in M3, M13, M92, M71, and $\omega$ Cen, mentioned in $\S$ Va, may disappear. This point will be taken up in a future paper.

M. AARONSON: Steward Observatory, University of Arizona, Tucson, AZ 85721

J. G. CoHEN: Department of Astronomy, Hale Observatories and California Institute of Technology, 105-24 Robinson, Pasadena, CA 91125

JAY A. Frogel: Cerro Tololo Inter-American Observatory, P.O. Box 26732, Tucson, AZ 85726

K. Matthews: Downs Laboratory of Physics, California Institute of Technology, Pasadena, CA 91125

S. E. Persson: Hale Observatories, 813 Santa Barbara Street, Pasadena, CA 91101 